\title{
Terrane correlation between Antarctica, Mozambique and Sri Lanka; comparisons of geochronology, lithology, structure and metamorphism and possible implications for the geology of southern Africa and Antarctica
}

\author{
G. H. GRANTHAM ${ }^{1}$, P. H. MACEY ${ }^{2}$, B. A. INGRAM ${ }^{1}$, M. P. ROBERTS ${ }^{3,4}$ \\ R. A. ARMSTRONG ${ }^{5}$, T. HOKADA ${ }^{6}$, K. SHIRAISHI ${ }^{6}$, C. JACKSON ${ }^{7}$, \\ A. BISNATH ${ }^{8} \&$ V. MANHICA ${ }^{9}$ \\ ${ }^{1}$ Council for Geoscience, P/Bag X112, Pretoria, South Africa \\ (e-mail: grantham@geoscience.org.za) \\ ${ }^{2}$ Council for Geoscience, Bellville, South Africa \\ ${ }^{3}$ MSSP-Geomap Project, c/o Geological Survey of Papua New Guinea, \\ Port Moresby, Papua New Guinea \\ ${ }^{4}$ Council for Geoscience, Walmer, Port Elizabeth, South Africa \\ ${ }^{5}$ RSES, Australian National University, Canberra, A.C.T. 0200, Australia \\ ${ }^{6}$ National Institute of Polar Research, Itabashi, Tokyo, Japan \\ ${ }^{7} 51$ Saint David's Road, Claremont, Cape Town, South Africa \\ ${ }^{8}$ Caracle Creek International Consulting Inc., Johannesburg, South Africa \\ ${ }^{9}$ Direcão Nacional Geologia, Maputo, Mozambique
}

\begin{abstract}
Analysis of new lithological, structural, metamorphic and geochronological data from extensive mapping in Mozambique permits recognition of two distinct crustal blocks separated by the Lurio Belt shear zone. Extrapolation of the Mozambique data to adjacent areas in Sri Lanka and Dronning Maud Land, Antarctica permits the recognition of similar crustal blocks and allows the interpretation that the various blocks in Mozambique, Sri Lanka and Antarctica were once part of a mega-nappe, forming part of northern Gondwana, which was thrust-faulted $c$. $600 \mathrm{~km}$ over southern Gondwana during amalgamation of Gondwana at c. 590-550 Ma. The data suggest a deeper level of erosion in southern Africa compared with Antarctica. It is possible that this thrust domain extends, through the Zambezi Belt or Valley, as far west as the Damara Orogen in Namibia with the Naukluft nappes in Namibia, the Makuti Group, the Masoso Suite in the Rushinga area and the Urungwe klippen in northern Zimbabwe, fitting the mega-nappe pattern. Erosional products of the mountain belt are now represented by $700-400 \mathrm{Ma}$ age detrital zircons present in the various sandstone formations of the Transantarctic Mountains, their correlatives in Australia, as well as the Urfjell Group (western Dronning Maud Land) and probably the Natal Group in South Africa.
\end{abstract}

\begin{abstract}
'What's in a name? That which we call a rose by any other name would smell as sweet' (Romeo and Juliet (II, ii, 1-2), Shakespeare, $c$. 1595). This paper could equally have been titled 'The nature and extent of the Lurio Belt inferred from the geochronology, structure, lithology and metamorphic histories of adjacent crustal blocks' or alternatively 'The errant hitchhiker terranes of northern Gondwana'
\end{abstract}

In 2000 an ambitious project to map Mozambique was initiated by the Mozambique Government The project was funded by the World Bank, the Nordic Development Fund and the governments of South Africa and Mozambique. The Norwegian Geological Survey (NGU) and British Geological Survey (BGS) consortium assumed responsibility for mapping most of northeastern Mozambique (Fig. 1). A consortium including the Finnish Geological Survey (GTK) and a private company assumed responsibility for most of northwestern, central and southern Mozambique (Fig. 1) whereas the Council for Geoscience of South Africa assumed responsibility for the mapping of eleven $1 \times 1$ degree sheets (Fig. 1). Nine of these 


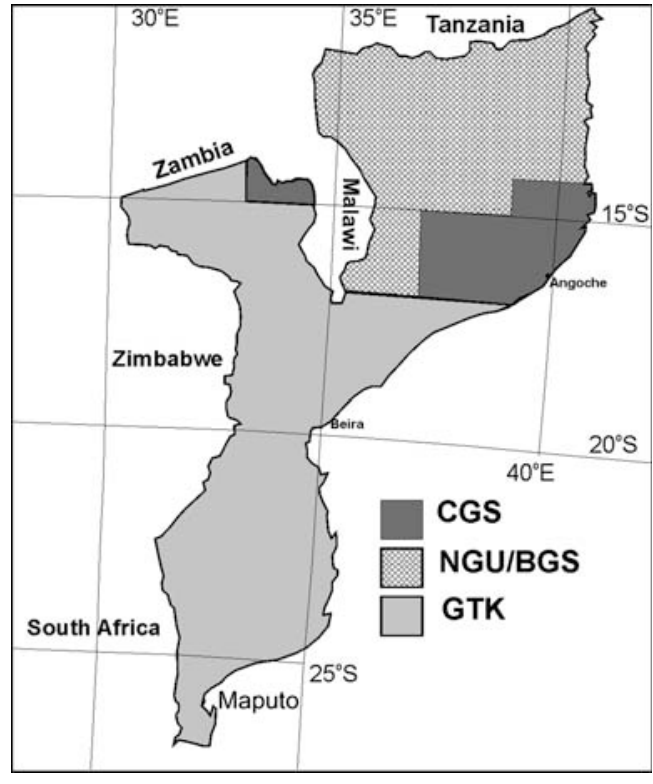

Fig. 1. Map of Mozambique showing the areas mapped by the various groups. CGS, Council for Geoscience of South Africa; NGU/BGS, Norwegian Geological Survey-British Geological Survey; GTK, Finnish Geological Survey.

sheets were located in NE Mozambique and two in NW Mozambique. The funding for mapping of the areas of northern Mozambique mapped by the NGU-BGS and GTK teams came from the Nordic Development Fund.

The project is now complete and the potential implications of some of the new data gathered in the mapping exercise are presented in summarized form and interpreted here. A review of the basement geology of Mozambique (Grantham et al. 2003) highlighted the paucity of reliable geochronological data in Mozambique itself (approximately five single zircon determinations at the time), in contrast to neighbouring areas. It also highlighted that a potential crustal boundary existed between the apparently juvenile southern end of the Mozambique Belt and an older block in northern Mozambique, with the northern end having limited juvenile rocks with extensive reworking of Palaeoproterozoic and Archaean rocks. The current project along with other studies has resulted in the number of new, reliable zircon ages now exceeding 100. Some of these data are summarized here and interpreted along with data from surrounding areas in a Gondwana context. These data have been critical for the definition of the model presented here. Recognizing the reconnaissance nature of the mapping, the application of low- to high-resolution magnetic and radiometric aerial surveys and Landsat data has also contributed significantly to the new interpretation presented here by confirming the Lurio Belt as a prominent geophysical and geological structure. The Lurio Belt is manifested in the field as a north to $\mathrm{NW}$, intermediate to steeply dipping, zone of high strain. The more exact nature of the Lurio Belt (i.e. thrust zone, strike-slip zone, zone of pure shear?) is the subject of current debate and has not been completely resolved by the mapping programme in Mozambique.

The Lurio Belt (LB) was first described by Jourde \& Vialette (1980), who described it as a suture of a major Lurian orogen with diverging nappes to the north and south (Pinna et al. 1993). Later interpretations by Pinna and others (Pinna 1995) considered it to be a late southerly thrust synform. Pinna et al. (1993) and Sacchi et al. (2000) proposed extensions of the Lurio structure into the Zambezi Belt, both of which involved late south-directed reverse thrusting.

Jamal (2005) described a complex history for the Lurio Belt, reporting that it has been affected by four phases of deformation under granulite- to amphibolite-facies conditions. The history recorded by Jamal (2005) is summarized as follows. An earlier deformation $\left(D_{1}\right)$ along the belt is represented by felsic segregations that commonly trace the $\mathrm{D}_{2} / \mathrm{F}_{2}$ folds. Map- and outcrop-scale $\mathrm{F}_{2}$ $\mathrm{NE}-\mathrm{SW}$-oriented isoclinal folds generally have subhorizontal fold axes. Fold attitudes are described as varying from subvertical upright to NW-dipping. Associated fold asymmetry suggests that these structures represent SE-directed thrusting. Continuous NW-SE shortening, with a dextral shear component, led to the refolding of the $\mathrm{D}_{2}$ isoclines about the $\mathrm{F}_{3}$ open to tight subvertical folds and the formation of an $S_{3}$ axial-plane cleavage. $D_{3}$ is interpreted as involving strike-slip shear under amphibolite-facies $P-T$ conditions. $\mathrm{S}-\mathrm{C}^{\prime}$ mylonitic fabrics observed throughout the Lurio Belt suggest a transpressive regime in response to an oblique compression. Thus the NE-SW Lurio Belt probably acted as a shear zone that accommodated high strain during a collision event that affected NE Mozambique.

Viola et al. (2006) have recently questioned the interpretation of the Lurio Belt as a major suture. They reported that structures along the belt vary greatly, involving intense linear structures in the $\mathrm{NE}$, becoming wider and less belt-like in the SW. They described tight to isoclinal folds with NNW-dipping axial planes and roughly down-dip stretching lineations. No clear kinematic indicators were observed. Strain accommodation, involving folding and conjugate shear zones within the Lurio Belt, is more intense than in the surrounding rocks. Evidence of SSE-NNW-directed regional 
compression is pervasive. Viola et al. concluded that the Lurio Belt represents a belt of repeated activity and reworking and that the last strain increment reflects pure shear bulk flattening of the belt, lacking significant regional belt parallel simple shear. In contrast to the south-directed transport direction, they have inferred extensional collapse toward the WNW.

Various workers have proposed that the Lurio Belt extends into Sri Lanka (Kröner 1991, 2001; Grantham et al. 2003) and is represented there by the shear zone separating the Highland Complex from the Vijayan Complex. This correlation is supported by the proximity of Sri Lanka to northern Mozambique suggested by various Gondwana reconstructions (Lawver et al. 1998; Reeves \& de Wit 2000).

This paper summarizes lithological, geochronological, structural and metamorphic data and interprets them to suggest that the Lurio Belt represents a deep-crustal terrane boundary in northern Mozambique and that its possible extensions into Sri Lanka and Dronning Maud Land, Antarctica, as a low-angle thrust nappe complex, permit the recognition of various crustal blocks or terranes separated by correlatives of the Lurio Belt in varying attitudes.

\section{Crustal structure of Mozambique}

Interpretation of aeroradiometric and aeromagnetic data supported by reconnaissance ground mapping by the various mapping teams has facilitated the recognition that NE Mozambique is divided into two dominant blocks separated by the Lurio Belt (Fig. 2) and its possible extensions westwards. For the purposes of this paper the block north of the Lurio Belt is termed the Namuno Block and that south of the Lurio Belt the Nampula Block.

The Namuno Block comprises an accretionary stack of thrust-faulted complexes (Fig. 1) of varying age (Bingen et al. 2006; Bjerkgard et al. 2006; Viola et al. 2006). Mesoproterozoic complexes include the Unango, Marrupo, Naroto, Meluco and Angonia Complexes whereas Neoproterozoic complexes include the Xixano, Montepuez, Lalamo, M'Sawize and Muaquia Complexes (Bingen et al. 2006; Hollick et al. 2006; Thomas et al. 2006; Viola et al. 2006; Grantham et al. 2007a; Fig. 2). The thrust-faulted accretionary stack defined by these workers can be extended further west, via Malawi, to northwestern Mozambique, with Mesoproterozoic rocks of the Southern Irumide Belt forming the footwall to similar-age high-grade gneisses thrust westwards (Grantham et al. 2007a). The Southern Irumide Belt is similarly interpreted to be underlain by late Palaeoproterozoic basement intruded by continental margin arc-related magmas between 1.09 and 1.4 Ga and strongly overprinted during the Pan-African Orogen (Johnson et al. 2006). The Southern Irumide Belt is also interpreted to comprise four shear zone bounded terranes, with the bounding faults having NW-SE-oriented strikes (Johnson et al. 2006). The Namuno Block thrust stacks recognized by Bingen et al. (2006) and Viola et al. (2006) and the Angonia Complex (Grantham et al. 2007a) are reported to have involved top-to-the-west and -WNW deformation whereas those further west are inferred to involve top-to-the-SW orientation (Johnson et al. 2006). It is readily apparent from the geophysical data that the whole thrust stack comprising the Mesoproterozoic to Neoproterozoic rocks from the Mozambique coast to the Angonia Complex in the west is itself sheared and rotated, with an apparent sinistral sense of rotation, where these rocks merge with the Lurio Belt and its possible extensions in the south. This indicates that the intense ductile strain deformation recorded in the north- to NW-dipping Lurio Belt either post-dated the amalgamation of the various complexes in the Namuno Block or was part of a synchronous, larger-scale, sinistral transpressional structure, with the ENEWSW-oriented Lurio Belt being the central main shear zone bounded on the north by a westward (sinistral) directed accretionary stack.

In contrast, the radiometric and aeromagnetic data for the area south of the Lurio Belt, the Nampula Block, do not show the same accretionary stack configuration. The Nampula Block is dominated by medium-grade migmatitic tonalitic orthogneisses and paragneisses and quartzofeldspathic orthogneisses that are complexly interfolded and intruded by undeformed to locally weakly deformed granitic intrusions. At least two granulite-grade klippen, the Monapo Complex and the Mugeba Complex (Pinna et al. 1993) are recognized overlying the Nampula Block. These two klippen contain high-grade granulite ortho- and paragneisses and have been regarded as remnants of a larger thrust sheet or sheets (Pinna et al. 1993). The granulite-grade klippen suggested by Pinna et al. (1993) in the vicinity of Nampula is not a klippen, but rather an area of sporadic in situ charnockitization that probably developed through the action of late-tectonic fluids (Macey et al. 2007). The Nampula Block is also partially transgressed by the north-south-oriented mylonitic Namama sinistral strike-slip shear zone (Cadoppi et al. 1987) in the SW (Fig. 2). The Namama Shear Zone appears to curve and disappear into $\mathrm{NE}-\mathrm{SW}$-oriented layer-parallel structures at its northern end.

The lack of continuity of the Namuno Block nappe complex across the Lurio Belt implies that 


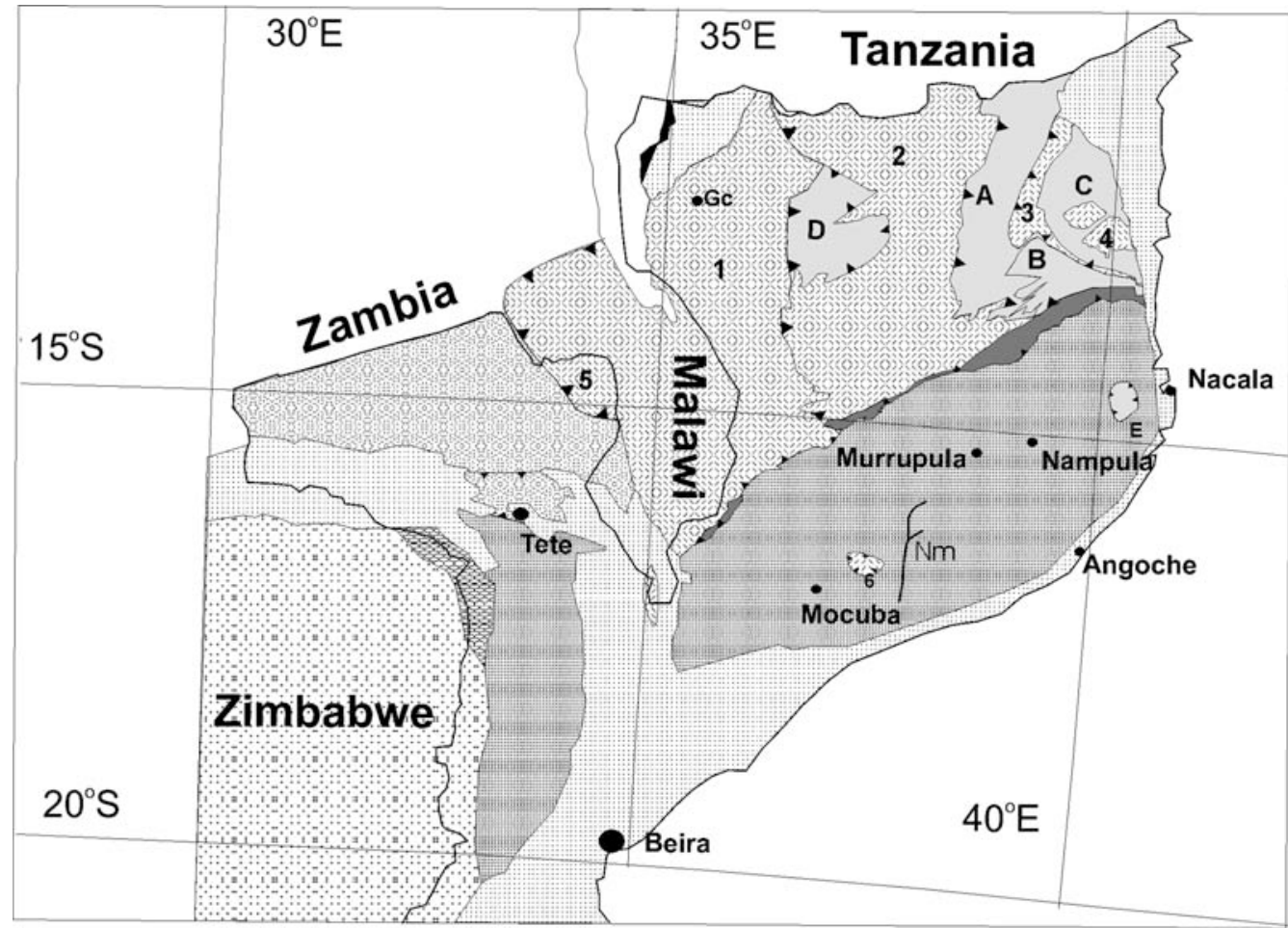

Post Palaeozoic cover

\section{Ocua Complex}

Xixano(A), Montepuez(B)

Lalamo (C) M'Sizwe + Muaquia(D) Monapo (E) Complexes

Unango(1) Marrupo(2) Naruto(3) Maluco(4) Angonia (5) Mugeba (6) Complexes

Tete and Southern Irumide Mesoproterozoic Complexes

Nampula Sub Province

Mesoproterozoic

Neoproterozoic

Neoproterozoic

Mesoproterozoic
Ponte Messuli Complex

Zimbabwe

Craton + Cratonic cover
Archaean + Paleo- to + Mesoproterozoic

Fig. 2. Map showing the crustal structure of northern Mozambique and adjacent areas. The location of the sinistral Namama Shear Zone (Nm) is shown east of Mocuba in the south of the Nampula Block and the approximate location of the Geci Group (Gc) is shown in far northern Mozambique east of Malawi. The map is compiled from Barr \& Brown (1987), Bingen et al. (2006), Bjerkgard et al. (2006), Hollick et al. (2006), Thomas et al. (2006), Grantham et al. $(2007 a, b)$ and Macey et al. (2007).

the Lurio Belt is not just a crustal shear zone that has segmented a uniform block of crust but represents a fundamental boundary along which two different blocks have been juxtaposed. The exact location of extensions of the Lurio structure westwards through southern Malawi into NW Mozambique and on into the Zambezi Valley are less clear, as a result of much of the area being overlain by Karoo-age sedimentary and volcanic rocks as well as being transected by the southern extensions of the western limb of the East
African Rift. Pinna et al. (1993) Pinna (1995), Sacchi et al. (2000) and Grantham et al. (2003) have supported an extension of the Lurio Belt into the Zambezi Belt.

During the current mapping programme it was recognized by Koistinen et al. (2006) and Westerhof (2006) that the Tete Complex is allochthonous and forms the hanging wall of a large southward-directed thrust fault. These workers also record a large southward-directed $c$. east-west shear zone $c .10 \mathrm{~km}$ south of Tete. Barr \& Brown 
(1987) also reported a major east-west-oriented shear zone, the Sanangoe thrust zone $c .40 \mathrm{~km}$ north of Tete with a top-to-the-north direction of transport. It is possible that these structures represent extensions of the Lurio Belt westwards. In conclusion, the mapping programme has facilitated the definition of the Lurio Belt as a major crustal boundary, which will be used to interpret the geochronological, structural and lithological variations described below.

\section{Rock types}

\section{Namuno Block}

The composition of the Unango Complex varies widely and consists of granitic gneisses, some locally charnockitic, with biotite-hornblende gneisses and quartzite. The metamorphic grade varies from amphibolite to granulite grade and the rocks are extensively migmatized (Bjerkgard et al. 2006).

Aeroradiometric and aeromagnetic surveys suggest that the rocks of the Unango Complex continue southwestwards through Malawi and become the supracrustal Angonia Complex in NW Mozambique west of the Malawi border. In this area interlayered, dominantly quartzofeldspathic gneisses with subordinate tonalitic and metabasic gneisses have been reported (Grantham et al. 2007a). Rare metapelitic gneisses and marbles are also seen. The metabasic gneisses have chemistry typical of enriched mid-ocean ridge basalt (E-MORB) rocks (Grantham et al. 2007a). Intruded into the supracrustal rocks are monzonites, syenites, anorthosite and pyroxenites of mostly uncertain age. The supracrustal sequences have ages of $c$. 1100-1050 Ma whereas an undeformed to weakly deformed monzonite has been dated at c. $560 \mathrm{Ma}$ (Grantham et al. 2007a). Metamorphic overprints dated at $c$. $550 \mathrm{Ma}$ have been reported from zircon rims and metamorphic titanite (Grantham et al. 2007a). The Angonia Group gneisses are thrust-faulted over granites of the Southern Irumide Complex to the east (Grantham et al. 2007a).

The Marrupa Complex is dominated by granitic to tonalitic gneiss, with mafic amphibolitic orthogneisses, quartzite and quartz-feldspar gneiss (Bjerkgard et al. 2006). The rocks are characterized by amphibolite-facies mineralogy. The geochemistry of the orthogneisses suggests that they are mediumto high-K calc-alkaline rocks with $\mathrm{SiO}_{2}$ ranging between $c .42$ and $78 \mathrm{wt} \%$ and $\mathrm{K}_{2} \mathrm{O}$ ranging between 0.3 and $6.1 \mathrm{wt} \%$ (Bjerkgard et al. 2006). The Nairoto Complex consists of variably migmatized granitic to tonalitic orthogneisses with calc-alkaline compositions (Bjerkgard et al. 2006). Mineral assemblages are typical of amphibolite-facies metamorphism (Bjerkgard et al. 2006).

The Meluco Complex comprises mostly granitic to granodioritic orthogneisses. Mineral assemblages are typical of amphibolite-facies metamorphism (Bjerkgard et al. 2006). The Xixano Complex includes part of the Chiure Supergroup and autochthonous supracrustal gneisses described by Pinna et al. (1993), and comprises a variety of paragneisses including marble, biotite gneiss, mica schists, meta-arenites, granitic to tonalitic gneisses and amphibolites (Bjerkgard et al. 2006). The metamorphic grade within the Xixano Complex is amphibolite facies to granulite facies (Bjerkgard et al. 2006).

The Muaquia Complex comprises granitic, tonalitic and gabbroic gneisses, amphibolites, mica gneiss and calc-silicate gneisses, and is predominantly mafic to intermediate in composition (Bjerkgard et al. 2006). The rocks have mineralogy typical of dominantly amphibolite-facies metamorphism (Bjerkgard et al. 2006). The M'Sawize Complex comprises banded migmatitic gneisses, granulitic gneiss and mafic granulite with mineralogy typical of granulitefacies metamorphism (Bjerkgard et al. 2006). The M'Sawize Complex comprises part of the Msawize Group of Pinna et al. (1993), who included their unit as part of the Lurio Supergroup.

The Lalamo Complex contains a variety of paragneisses including marble, biotite gneiss, mica schists, meta-arenites and granitoid gneisses with mineralogy typical of amphibolite-facies metamorphism (Bjerkgard et al. 2006). The Montepuez Complex was previously defined as part of the large Chiure Group by Pinna et al. (1993) and contains granitic to granodioritic gneiss, biotite gneiss and marbles with mineral parageneses typical of amphibolite-facies metamorphism (Bjerkgard et al. 2006). The Ocua Complex comprises rocks defined as the Lurio Supergroup by Pinna et al. (1993) and consists of a tectonic mélange (Thomas et al. 2006). The main rock types are mostly granulitic gneisses of tonalitic, dioritic and granitic composition, amphibolitic and granulitic gneisses as well as ultramafic and metaluminous gneiss (Bjerkgard et al. 2006). The high strains characteristic of the eastern Lurio Belt become less distinct to the SW (Viola et al. 2006).

\section{Nampula Block}

The description of the rock units of the Nampula Block is summarized from Macey et al. (2007), and Grantham et al. (2007b), who confirmed descriptions by earlier workers including Pinna et al. (1993) and Sacchi et al. (1984) amongst others. Six lithostratigraphic units are recognized in the Nampula Block. They comprise the Mesoproterozoic gneisses of the Mocuba Suite, the Culicui 
Suite, the Rapale Gneiss, the Mamala Gneiss, the Molocue Group and the Cambrian granites of the Murrupula Suite. The Mocuba Suite consists dominantly of migmatitic banded tonalitic gneisses and subordinate mafic rocks with amphibolitefacies mineralogy. Compositions are dominantly calc-alkaline. Crystallization ages of $c .1125 \mathrm{Ma}$ have been recorded (Macey et al. 2007). Not only does the strongly migmatized character of the Mocuba Suite distinguish it from the other Mesoproterozoic gneisses, it also indicates that a Mesoproterozoic orogenic event was experienced by these rocks of the Nampula Block. The Rapale Gneiss is of similar tonalitic composition but is clearly intrusive into the Mocuba Suite and has crystallization ages of c. $1090 \mathrm{Ma}$. The Culicui Suite is dominated by megacrystic, typically highly sheared, augen gneisses, which locally, in low-strain zones, preserve charnockitic mineralogy. In general, however, the metamorphic assemblages are typical of amphibolite-facies metamorphism. Crystallization ages from the Culicui Suite range typically between c. 1070 and $1090 \mathrm{Ma}$. The Mamala Gneiss is relatively uniform equigranular medium- to fine-grained leuco-quartzofeldspathic gneiss with uniform field and geophysical characteristics. Crystallization ages from the Mamala Gneiss are c. 1090 Ma. The Molocue Group comprises a banded interlayered sequence of dominantly quartzo-feldspathic para- and orthogneisses with subordinate amphibolites and calc-silicates.

Three younger pre-Gondwana breakup lithological entities are recognized in the Nampula Block: the Murrupula Suite, the Mugeba Complex and the Monapo Complex. The Mugeba Complex is dominated by granulite-grade garnet-pyroxene intermediate orthogneisses with subordinate garnetpyroxene metabasic granulites and garnet-sillimanite-rutile metapelitic gneiss. The Monapo Complex contains mostly granulite-grade banded supracrustal gneisses with subordinate Grt-Sil-Rtbearing metapelites. Intruded into the supracrustal gneisses are weakly deformed to apparently undeformed clinopyroxenites, syenites, granite and carbonatite (Siegfried 1999; Grantham et al. 2007b). Crystallization ages of c. $635 \mathrm{Ma}$ are recognized from the Monapo Complex (Jamal 2005; Grantham et al. 2007b), whereas discordant zircons from the Mugeba Complex suggest a $c .1000$ Ma protolith. Metamorphic ages from these complexes are $c$. $635 \mathrm{Ma}$ and c. $580 \mathrm{Ma}$ (Grantham et al. 2007b; Macey et al. 2007).

The Murrupula Suite comprises granitoid intrusions, mostly undeformed and emplaced as kilometre-scale plutons to metre-scale pegmatitic dykes (Macey et al. 2007). The compositions vary from syenitic to granitic and include equigranular medium-grained varieties to coarse-grained porphyritic types. The chemistry of the intrusions varies from metaluminous A-type rocks to peraluminous mica granites (Macey et al. 2007). The A-type intrusions have chemistries typical of $\mathrm{A}_{2}$ granites (Eby 1992), which are interpreted as typically being generated post-orogenically (Bonin 2007) and from continental crust that has been through a cycle of continent-continent collision or island arc magmatism (Eby 1992). Crystallization ages of the intrusions vary from c. 495 to $c .530 \mathrm{Ma}$.

\section{Discussion}

On a purely lithological composition basis there is little to indicate a major crustal boundary defined by the Lurio Belt. Broadly, the Namuno Block and the Nampula Block are dominantly underlain by quartzofeldspathic gneisses, with the Lurio Belt and related Ocua Complex rocks being characterized by strong geophysical signatures and evidence of high strain in the field. The reduced geophysical signature of the Lurio Belt in the SW could possibly result from the structure attaining a shallower dip and being folded. This possibility requires additional investigation.

Lithological distinguishing factors between the Namuno and Nampula Blocks include (1) a higher prevalence of supracrustal rocks containing metapelites and marbles north of the Lurio Belt as well as (2) the subordinate, but significant, presence of relatively alkaline syenitic orthogneisses north of the Lurio Belt. The only lithological exceptions to these distinguishing factors are found in the Monapo and Mugeba Complex klippen overlying the Nampula Block.

\section{Geochronology of Mozambique and surrounding areas}

In comparing the geochronology of the different areas, histograms with bin sizes of $50 \mathrm{Ma}$ are used in conjunction with probability density distributions. The limitations of these two methods have been described in detail by Sircombe (2000). The limitation of the use of histograms alone is that they do not take the error estimate of an age into account whereas a limitation in the use of probability density distributions alone is that they do not quantify the number of ages recorded within a particular age or bin range.

\section{Southeastern Africa}

Figure 3 shows igneous crystallization and metamorphic ages from the Namuno Block, comprising the area north of the Lurio Belt in NE Mozambique 

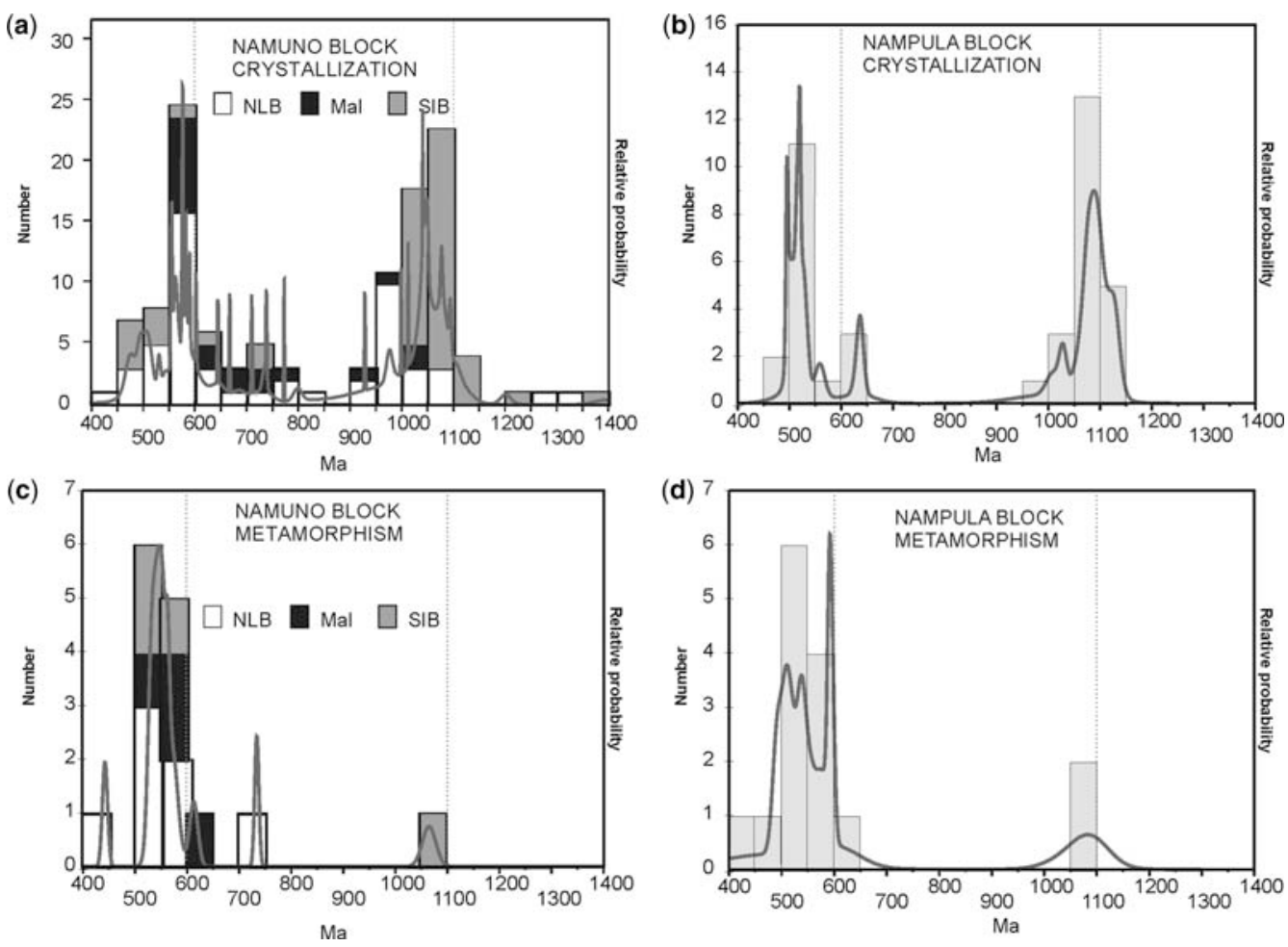

Fig. 3. (a, b) Histograms and probability density curves of crystallization ages from the Namuno Block (a), comprising an area north of the Lurio Block (NLB), a southern Irumide Belt Block (SIB) and Malawi (Mal), and (b) Nampula Block. (c, d) Histograms and probability density curves of metamorphic ages from the Namuno Block (c), comprising an area north of the Lurio Block (NLB), a southern Irumide Belt Block (SIB) and Malawi (Mal), and (d) Nampula Block igneous crystallization and metamorphic zircons. The lines for $600 \mathrm{Ma}$ and $1100 \mathrm{Ma}$ are shown for reference in all the figures.

(NLB), Malawi (Mal) and the southern Irumide areas of southern Zambia and NW Mozambique (SIB) and the Nampula Block (see Fig. 2).

Data available include those generated during the mapping programme (Bingen et al. 2006; Mänttärri et al. 2006; Grantham et al. 2007a, b; Macey et al. 2007) along with additional data from Costa et al. (1994), Kröner et al. (1997, 2001), Sacchi et al. (2000), Manhica et al. (2001), Jamal (2005) and Johnson et al. (2005, 2006). The data are dominantly derived from sensitive high-resolution ion microprobe (SHRIMP), inductively coupled plasma mass spectrometry (ICP-MS) or thermal ionization mass spectrometry (TIMS) analyses, except for the $\mathrm{Pb}-\mathrm{Pb}$ evaporation data of Kroner et al. (2001) from southern Malawi. A few mineral-whole-rock $\mathrm{Sm}-\mathrm{Nd}$ data are included from the Southern Irumide Block. In addition, only the data from the Southern Irumide Belt of Johnson et al. $(2005,2006)$ have been used for the geochronological comparisons below. The data used for the crystallization age analysis from the Namuno Block are shown in Table 1 whereas the age data from the Nampula Block are summarized in Table 2.

Comparison of the histograms and probability density distribution curves shows that all areas have broadly extensive Mesoproterozoic crystallization ages of $c$. $900-1150 \mathrm{Ma}$ as well as Neoproterozoic-Cambrian ages of c. 650-450 Ma. However, a significant difference is that the Namuno Block (Fig. 3a) is characterized by crystallization ages from c. 650 to $900 \mathrm{Ma}$, which are absent from the Nampula Block (Fig. 3b). In the Nampula Block three ages of $c .630 \mathrm{Ma}$ are recorded from the Monapo (two) and Mugeba klippen (Jamal 2005; Grantham et al. 2007b; Macey et al. 2007). These data clearly demonstrate that the Monapo and Mugeba klippen have age characteristics similar to rocks exposed north of the Lurio Belt in the Namuno Block. Other rock types with ages of c. 800 Ma possibly located south of Lurio Belt extensions are the granitic rocks intruded into the Rushinga area of NE Zimbabwe and western Mozambique 
Table 1. Crystallization age data used to construct figures for the Namuno Block from the southern Irumide Belt and Malawi

\begin{tabular}{|c|c|c|c|c|c|}
\hline Unit and sample number & Method & Age (Ma) & Error & Block & Source \\
\hline $\begin{array}{l}\text { Biotite-hornblende gneiss } \\
\text { (MA16) }\end{array}$ & SHRIMP & 664 & 27 & S. Malawi & Kröner et al. (2001) \\
\hline Pelitic paragneiss (MA8) & SHRIMP & 576 & 11 & S. Malawi & Kröner et al. (2001) \\
\hline $\begin{array}{l}\text { Chewore Ophiolite plagiogranite } \\
\text { (sample SJ106.1) }\end{array}$ & SHRIMP & 1393 & 22 & S. Irumide Belt & Johnson et al. (2005) \\
\hline $\begin{array}{l}\text { Kaourera Arc meta-dacite } \\
\text { (sample SJ220) }\end{array}$ & SHRIMP & 1082 & 7 & S. Irumide Belt & Johnson et al. (2005) \\
\hline Kadunguri Whiteschists & SHRIMP & 1066 & 21 & S. Irumide Belt & Johnson et al. (2005) \\
\hline $\begin{array}{l}\text { Chewore Inlier Granulite } \\
\text { Terrane (sample ADC) }\end{array}$ & SHRIMP & 1071 & 8 & S. Irumide Belt & Johnson et al. (2005) \\
\hline $\begin{array}{l}\text { Chewore Inlier Zambezi Terrane } \\
\text { orthogneiss (sample AF) }\end{array}$ & SHRIMP & 1083 & 8 & S. Irumide Belt & Johnson et al. (2005) \\
\hline $\begin{array}{l}\text { ZM007 meta-dacite Chongwe } \\
\text { River }\end{array}$ & SHRIMP & 1088 & 20 & S. Irumide Belt & Johnson et al. (2005) \\
\hline $\begin{array}{l}\text { CH6 banded mafic gneiss } \\
\text { Chowe River }\end{array}$ & SHRIMP & 1051 & 12 & S. Irumide Belt & Johnson et al. (2005) \\
\hline CH7 meta-tuff Chowe River & SHRIMP & 1064 & 15 & S. Irumide Belt & Johnson et al. (2005) \\
\hline CH7 meta-tuff Chowe River & SHRIMP & 1037 & 8 & S. Irumide Belt & Johnson et al. (2005) \\
\hline $\mathrm{CH} 9$ meta-dacite Chowe River & SHRIMP & 1040 & 21 & S. Irumide Belt & Johnson et al. (2005) \\
\hline CH9 meta-dacite Chowe River & SHRIMP & 1105 & 22 & S. Irumide Belt & Johnson et al. (2005) \\
\hline $\begin{array}{l}\text { CH10 K-feldspar augen gneiss } \\
\text { Chowe River }\end{array}$ & SHRIMP & 1094 & 2 & S. Irumide Belt & Johnson et al. (2005) \\
\hline $\begin{array}{l}\text { CH10 K-feldspar augen gneiss } \\
\text { Chowe River }\end{array}$ & SHRIMP & 1105 & 9 & S. Irumide Belt & Johnson et al. (2005) \\
\hline $\begin{array}{l}\text { Charnockite associated with } \\
\text { Chipera gabbro-anorthosite }\end{array}$ & TIMS & 1050 & 20 & S. Irumide Belt & Johnson et al. (2005) \\
\hline $\begin{array}{l}\text { Garnet-spinel-cordierite gneiss } \\
\text { (Chipata Gneiss) }\end{array}$ & TIMS & 1046 & 3 & S. Irumide Belt & Johnson et al. (2005) \\
\hline $\begin{array}{l}\text { Porphyritic granite (EP26 } \\
\text { Petauke-Sinda Terrane) }\end{array}$ & LA-ICP-MS & 1125 & 15 & S. Irumide Belt & Johnson et al. (2005) \\
\hline $\begin{array}{l}\text { Deformed K-feldspar augen } \\
\text { gneiss CHP2a }\end{array}$ & SHRIMP & 1046 & 4 & S. Irumide Belt & Johnson et al. (2006) \\
\hline Undeformed syenite CHP2c & SHRIMP & 1050 & 7 & S. Irumide Belt & Johnson et al. (2006) \\
\hline $\begin{array}{l}\text { Moderately deformed coarse- } \\
\text { grained syenite CHP3 }\end{array}$ & SHRIMP & 543 & 6 & S. Irumide Belt & Johnson et al. (2006) \\
\hline $\begin{array}{l}\text { Opx-bearing granulite from } \\
\text { Madzimoyo quarry CHP4a }\end{array}$ & SHRIMP & 1076 & 6 & S. Irumide Belt & Johnson et al. (2006) \\
\hline $\begin{array}{l}\text { Garnet-opx-bearing mafic layer } \\
\text { Madzimoyo quarry CHP4b }\end{array}$ & SHRIMP & 1977 & 11 & S. Irumide Belt & Johnson et al. (2006) \\
\hline $\begin{array}{l}\text { Opx-bearing granulite roadside } \\
\text { Madzimoyo quarry CHP5 }\end{array}$ & SHRIMP & 1047 & 20 & S. Irumide Belt & Johnson et al. (2006) \\
\hline $\begin{array}{l}\text { Coarse-grained hbl-biotite } \\
\text { equigranular granite CHP6a }\end{array}$ & SHRIMP & 1038 & 6 & S. Irumide Belt & Johnson et al. (2006) \\
\hline $\begin{array}{l}\text { Coarse-grained K-feldspar } \\
\text { porphyritic granite CHP8 }\end{array}$ & SHRIMP & 1061 & 13 & S. Irumide Belt & Johnson et al. (2006) \\
\hline $\begin{array}{l}\text { Coarse-grained K-feldspar } \\
\text { porphyritic granite CHP10 }\end{array}$ & SHRIMP & 1076 & 14 & S. Irumide Belt & Johnson et al. (2006) \\
\hline $\begin{array}{l}\text { Foliated or banded qtz-feldspar } \\
\text { migmatite, leucosome portion } \\
\text { CHP11a }\end{array}$ & SHRIMP & 1950 & 67 & S. Irumide Belt & Johnson et al. (2006) \\
\hline $\begin{array}{l}\text { K-feldspar porphyritic granite } \\
\text { CHP12 }\end{array}$ & SHRIMP & 1038 & 9 & S. Irumide Belt & Johnson et al. (2006) \\
\hline $\begin{array}{l}\text { K-feldspar porphyritic granite } \\
\text { CHP13 }\end{array}$ & SHRIMP & 1058 & 34 & S. Irumide Belt & Johnson et al. (2006) \\
\hline $\begin{array}{l}\text { Magmatically banded K-feldspar } \\
\text { porphyritic granite PS } 17\end{array}$ & SHRIMP & 479 & 9 & S. Irumide Belt & Johnson et al. (2006) \\
\hline $\begin{array}{l}\text { Coarse-grained bt-poor qtz-plag } \\
\text { granite PS18 }\end{array}$ & SHRIMP & 510 & 6 & S. Irumide Belt & Johnson et al. (2006) \\
\hline
\end{tabular}


Table 1. Continued

\begin{tabular}{|c|c|c|c|c|c|}
\hline Unit and sample number & Method & Age (Ma) & Error & Block & Source \\
\hline $\begin{array}{l}\text { Fine-grained magmatically } \\
\text { banded syenite PS19 }\end{array}$ & SHRIMP & 494 & 5 & S. Irumide Belt & Johnson et al. (2006) \\
\hline $\begin{array}{l}\text { Medium-grained, equigranular } \\
\text { qtz-plag syenite PS } 28\end{array}$ & SHRIMP & 495 & 10 & S. Irumide Belt & Johnson et al. (2006) \\
\hline $\begin{array}{l}\text { Undeformed equigranular coarse } \\
\text { qtz-Kfs-bt granite PS65 }\end{array}$ & SHRIMP & 1043 & 14 & S. Irumide Belt & Johnson et al. (2006) \\
\hline $\begin{array}{l}\text { Undeformed K-feldspar } \\
\text { porphyritic granite PS71b }\end{array}$ & SHRIMP & 720 & 12 & S. Irumide Belt & Johnson et al. (2006) \\
\hline $\begin{array}{l}\text { Patch equigranular granite in } \\
\text { coarse pegmatites PS73 }\end{array}$ & SHRIMP & 504 & 7 & S. Irumide Belt & Johnson et al. (2006) \\
\hline $\begin{array}{l}\text { Equigranular medium-grained } \\
\text { qtz-plag-bt granite PS76 }\end{array}$ & SHRIMP & 474 & 8 & S. Irumide Belt & Johnson et al. (2006) \\
\hline $\begin{array}{l}\text { Strongly deformed } \\
\text { quartzofeldspathic gneiss } \\
\text { PS78 }\end{array}$ & SHRIMP & 742 & 13 & S. Irumide Belt & Johnson et al. (2006) \\
\hline $\begin{array}{l}\text { Garnet-bearing pelitic migmatite } \\
\text { SZ16 }\end{array}$ & SHRIMP & 1984 & 21 & S. Irumide Belt & Johnson et al. (2006) \\
\hline $\begin{array}{l}\text { Deformed qtz-feld gneiss with } \\
\text { thin amphibolite SZ23 }\end{array}$ & SHRIMP & 1008 & 17 & S. Irumide Belt & Johnson et al. (2006) \\
\hline $\begin{array}{l}\text { Progressively mylonitized } \\
\text { porphyritic granite SZ25c }\end{array}$ & SHRIMP & 1023 & 12 & S. Irumide Belt & Johnson et al. (2006) \\
\hline $\begin{array}{l}\text { Strongly deformed } \\
\text { quartzofeldspathic gneiss } \\
\text { SZ26 }\end{array}$ & SHRIMP & 1961 & 31 & S. Irumide Belt & Johnson et al. (2006) \\
\hline $\begin{array}{l}\text { Strongly deformed hornblende- } \\
\text { biotite gneiss SZ27 }\end{array}$ & SHRIMP & 647 & 11 & S. Irumide Belt & Johnson et al. (2006) \\
\hline $\begin{array}{l}\text { Tonalite Angonia Complex } \\
\text { GGZ238 }\end{array}$ & SHRIMP & 1104 & 11 & S. Irumide Belt & Grantham et al. (2007a) \\
\hline $\begin{array}{l}\text { Metabasite Angonia Complex } \\
\text { GGZ229 }\end{array}$ & SHRIMP & 1058 & 11 & S. Irumide Belt & Grantham et al. (2007a) \\
\hline Monzonite GGZ256 & SHRIMP & 568 & 5 & S. Irumide Belt & Grantham et al. (2007a) \\
\hline Desaranhama Granite & & 1041 & 4 & S. Irumide Belt & Mänttärri et al. (2006) \\
\hline Monte Capingo Suite & & 1201 & 10 & S. Irumide Belt & Mänttärri et al. (2006) \\
\hline Sinda granite & & 502 & 8 & S. Irumide Belt & Mänttärri et al. (2006) \\
\hline Monte Capirimpica Suite & & 1086 & 7 & S. Irumide Belt & Mänttärri et al. (2006) \\
\hline Cassacatiza Suite & & 1077 & 2 & S. Irumide Belt & Mänttärri et al. (2006) \\
\hline Monte Sanje Suite & & 1050 & 8 & S. Irumide Belt & Mänttärri et al. (2006) \\
\hline Granito Castanho & & 1050 & 2 & S. Irumide Belt & Mänttärri et al. (2006) \\
\hline Chipera Complex (Tete Suite) & $\mathrm{Sm}-\mathrm{Nd}$ & 1047 & 29 & S. Irumide Belt & Mänttärri et al. (2006) \\
\hline Macanga Granite & & 470 & 14 & S. Irumide Belt & Mänttärri et al. (2006) \\
\hline Mussata Granite & & 1046 & 20 & S. Irumide Belt & Mänttärri et al. (2006) \\
\hline Ocua Complex Charnockite & SHRIMP & 994 & 61 & N. of Lurio & Macey et al. (2007) \\
\hline $\begin{array}{l}\text { Marrupa Complex Tonalitic } \\
\text { Gneiss }\end{array}$ & SHRIMP & 951 & 44 & N. of Lurio & Grantham et al (2007b) \\
\hline Granitic gneiss (MA1) & $\mathrm{Pb} / \mathrm{Pb}$ evap. & 602.7 & 1 & Southern Malawi & Kröner et al. (2001) \\
\hline Diorite granulitic gneiss (MA2) & $\mathrm{Pb} / \mathrm{Pb}$ evap. & 644.9 & 0.9 & Southern Malawi & Kröner et al. (2001) \\
\hline Trondhjemite gneiss (MA3) & $\mathrm{Pb} / \mathrm{Pb}$ evap. & 582.9 & 1 & Southern Malawi & Kröner et al. (2001) \\
\hline Quartz monzonite gneiss (MA4) & $\mathrm{Pb} / \mathrm{Pb}$ evap. & 577.5 & 1 & Southern Malawi & Kröner et al. (2001) \\
\hline Charnockitic gneiss (MA6) & $\mathrm{Pb} / \mathrm{Pb}$ evap. & 590.5 & 1 & Southern Malawi & Kröner et al. (2001) \\
\hline Charnockitic gneiss(MA7) & $\mathrm{Pb} / \mathrm{Pb}$ evap. & 928.9 & 0.9 & Southern Malawi & Kröner et al. (2001) \\
\hline Pelitic paragneiss (MA8) & $\mathrm{Pb} / \mathrm{Pb}$ evap. & 576.7 & 1 & Southern Malawi & Kröner et al. (2001) \\
\hline Charnoenderbitic gneiss (MA9) & $\mathrm{Pb} / \mathrm{Pb}$ evap. & 1012.5 & 0.8 & Southern Malawi & Kröner et al. (2001) \\
\hline $\begin{array}{l}\text { Biotite-hornblende gneiss } \\
\text { (MA10) }\end{array}$ & $\mathrm{Pb} / \mathrm{Pb}$ evap. & 998.9 & 0.8 & Southern Malawi & Kröner et al. (2001) \\
\hline $\begin{array}{l}\text { Biotite-hornblende gneiss } \\
\text { (MA13) }\end{array}$ & $\mathrm{Pb} / \mathrm{Pb}$ evap. & 738.7 & 0.9 & Southern Malawi & Kröner et al. (2001) \\
\hline $\begin{array}{l}\text { Biotite-hornblende gneiss } \\
\text { (MA13) }\end{array}$ & $\mathrm{Pb} / \mathrm{Pb}$ evap. & 576.1 & 1 & Southern Malawi & Kröner et al. (2001) \\
\hline $\begin{array}{l}\text { Biotite-hornblende gneiss } \\
\text { (MA14) }\end{array}$ & $\mathrm{Pb} / \mathrm{Pb}$ evap. & 1040.6 & 0.7 & Southern Malawi & Kröner et al. (2001) \\
\hline
\end{tabular}


Table 1. Continued

\begin{tabular}{|c|c|c|c|c|c|}
\hline Unit and sample number & Method & Age (Ma) & Error & Block & Source \\
\hline Charnoenderbitic gneiss (MA15) & $\mathrm{Pb} / \mathrm{Pb}$ evap. & 554.7 & 1 & Southern Malawi & Kröner et al. (2001) \\
\hline $\begin{array}{l}\text { Biotite-hornblende gneiss } \\
\text { (MA16) }\end{array}$ & $\mathrm{Pb} / \mathrm{Pb}$ evap. & 667.5 & 0.9 & Southern Malawi & Kröner et al. (2001) \\
\hline Biotite gneiss (MA17) & $\mathrm{Pb} / \mathrm{Pb}$ evap. & 710.5 & 0.9 & Southern Malawi & Kröner et al. (2001) \\
\hline Biotite gneiss (MA17) & $\mathrm{Pb} / \mathrm{Pb}$ evap. & 556.1 & 1 & Southern Malawi & Kröner et al. (2001) \\
\hline Biotite gneiss (MA17) & $\mathrm{Pb} / \mathrm{Pb}$ evap. & 772.5 & 0.5 & Southern Malawi & Kröner et al. (2001) \\
\hline
\end{tabular}

The data from north of the Lurio Belt are from Jamal (2005) and Bingen et al. (2006).

Table 2. Crystallization ages from the Nampula Block

\begin{tabular}{|c|c|c|c|c|c|}
\hline $\begin{array}{l}\text { Rock type and sample } \\
\text { number }\end{array}$ & Unit & Method & Age (Ma) & Error & Source \\
\hline Granulite & Mocuba Complex & SHRIMP & 1028 & 7 & Costa et al. (1994) \\
\hline $\begin{array}{l}\text { Migmatitic granite } \\
\text { gneiss (sample MS5) }\end{array}$ & & SHRIMP & 1094 & 13 & Kröner et al. (2001) \\
\hline $\begin{array}{l}\text { Leucocratic granite } \\
\text { (sample MS6) }\end{array}$ & & SHRIMP & 1009 & 13 & Kröner et al. (2001) \\
\hline $\begin{array}{l}\text { Augen gneiss sample } \\
\text { NHF }\end{array}$ & $\begin{array}{l}\text { Nhansipfhe Megacrystic } \\
\text { Gneiss }\end{array}$ & SHRIMP & 1112 & 18 & Manhica et al. (2001) \\
\hline $\begin{array}{l}\text { Tonalitic gneiss sample } \\
\text { CVGN }\end{array}$ & $\begin{array}{l}\text { Chimoio Granodiorite } \\
\text { Gneiss }\end{array}$ & SHRIMP & 1108 & 12 & Manhica et al. (2001) \\
\hline Granite & Murrupula Suite & SHRIMP & 495 & 2 & Macey et al. (2007) \\
\hline Megacrystic charnockite & Culicui Suite & SHRIMP & 1074 & 13 & Macey et al. (2007) \\
\hline Augen gneiss & Culicui Suite & SHRIMP & 1082 & 26 & Macey et al. (2007) \\
\hline Tonalite & Mocuba Complex & SHRIMP & 1078 & 16 & Macey et al. (2007) \\
\hline Mocuba Suite & Mocuba Complex & SHRIMP & 1123 & 14 & Macey et al. (2007) \\
\hline Augen gneiss & Culicui Suite & SHRIMP & 1085 & 10 & Macey et al. (2007) \\
\hline Mocuba Gneiss & Mocuba Complex & SHRIMP & 1129 & 9 & Macey et al. (2007) \\
\hline Augen gneiss & Culicui Suite & SHRIMP & 1077 & 26 & Macey et al. (2007) \\
\hline Augen gneiss & Culicui Suite & SHRIMP & 1092 & 42 & Macey et al. (2007) \\
\hline Tonalitic gneiss & Rapale gneiss & SHRIMP & 1091 & 14 & Macey et al. (2007) \\
\hline Augen gneiss & Culicui Suite & SHRIMP & 1076 & 8 & Macey et al. (2007) \\
\hline Granitic gneiss & Mamala Gneis & SHRIMP & 1092 & 13 & Macey et al. (2007) \\
\hline Calc-silicate & Molucue Grp & SHRIMP & 1127 & 11 & Macey et al. (2007) \\
\hline Granite & Murrupula Suite & SHRIMP & 533 & 5 & Macey et al. (2007) \\
\hline Granitic gneiss & Molucue Grp & SHRIMP & 1090 & 22 & Macey et al. (2007) \\
\hline Granite & Murrupula Suite & SHRIMP & 504 & 12 & Macey et al. (2007) \\
\hline Augen gneiss & Culicui Suite & SHRIMP & 1073 & 16 & Macey et al. (2007) \\
\hline Tonalitic gneiss & Rapale gneiss & SHRIMP & 1095 & 8 & Macey et al. (2007) \\
\hline Granite & Murrupula Suite & SHRIMP & 521 & 4 & Macey et al. (2007) \\
\hline Syenite & Murrupula Suite & SHRIMP & 527 & 4 & Macey et al. (2007) \\
\hline Granite & Murrupula Suite & SHRIMP & 507 & 7 & Macey et al. (2007) \\
\hline Granite & Murrupula Suite & SHRIMP & 497 & 4 & Macey et al. (2007) \\
\hline Granite NB21-8 & Murrupula Suite & SHRIMP & 516 & 3 & Macey et al. (2007) \\
\hline Granite NB21-1 & Murrupula Suite & SHRIMP & 505 & 5 & Macey et al. (2007) \\
\hline Granite NB5-1 & Murrupula Suite & SHRIMP & 514 & 4 & Macey et al. (2007) \\
\hline Syenite & Ramiane Suite & SHRIMP & 634 & 8 & Grantham et al. $(2007 b)$ \\
\hline Granulite & Monapo Complex & SHRIMP & 637 & 6 & Grantham et al. (2007b) \\
\hline
\end{tabular}

Additional data are available from Jamal (2005). 
(Barton et al. 1993; Dirks et al. 1998; Vinyu et al. 1999; Hargrove et al. 2003; Fig. 2) as well as the Guro Bimodal Suite (Mänttäri et al. 2006; Westerhof 2006). These rocks are located along the margin of the Zimbabwe Craton and consequently their relationship to the Mozambique Belt is uncertain. Koistinen et al. (2006) related the c. $850 \mathrm{Ma}$ magmatism to extensional processes at the margin of the Zimbabwe Craton, whereas Westerhof (2006) suggested that these ages may be related to detachment thrusting. The $c .850 \mathrm{Ma}$ ages are therefore geographically restricted to areas at or close to the Zimbabwe Craton and consequently are anomalous in the Nampula Block.

The difference in ages between the Namuno and Nampula Blocks was recognized by Pinna (1995), although at that time the nature and origin of the age differences was unclear. Another important difference between the three areas is that the Nampula Block and southern Irumide area have some samples with crystallization ages between 1100 and $1200 \mathrm{Ma}$ whereas these ages are absent in the north of the Lurio Namuno area. Another difference is that the southern Irumide area appears to have dominantly $1000-1100 \mathrm{Ma}$ ages and fewer ages in the $<600 \mathrm{Ma}$ range. It is uncertain whether this is real or an artefact of sampling.

Metamorphic age data are summarized in Tables 3 (Namuno Block) and 4 (Nampula Block). Data sources include Kröner et al. (1997, 2001), Jamal (2005), Johnson et al. (2005, 2006), Bingen et al. (2006), Grantham et al. (2007a, b), and Macey et al. (2007). Comparison of the metamorphic ages shows that from the Namuno Block (Fig. 3c) no evidence of Mesoproterozoic metamorphism has been recorded north of the Lurio Belt, with metamorphic ages in the Namuno Block being c. 750$400 \mathrm{Ma}$ with 1050-1100 Ma metamorphism being recorded in the southern Irumide area. In contrast, data from the Nampula Block (Fig. 3d) indicate that metamorphism occurred during the Mesoproterozoic between 1050 and $1100 \mathrm{Ma}$ as well as during the time period c. 600-400 Ma.

In conclusion, from the histograms shown in Figure 3, it is recognized that the Namuno and Nampula Blocks have different characteristics, some fairly distinct (e.g. the common presence of 600-900 Ma ages in the Namuno Block and absence in the Nampula Block) and some subtle.

\section{Antarctica}

Extending the patterns recognized from the Namuno and Nampula blocks to the adjacent areas of Dronning Maud Land (DML), Antarctica, in a Gondwana context (Fig. 4), the following aspects become apparent. The age distribution for the Nampula Block is virtually identical to that observed in western DML (Sverdrupfjella + Kirwanveggean) (Fig. 5a) with crystallization ages in both areas falling in the time periods 950-1200 Ma and 470-500 Ma and metamorphic ages being recorded for the periods $950-1100 \mathrm{Ma}$ and $450-600 \mathrm{Ma}$ (Fig. 5b). The data utilized in Figure $5 \mathrm{a}$ and $\mathrm{b}$ are summarized in Tables 5 and 6 with the data derived from Harris et al. (1995), Krynauw \& Jackson (1996), Jackson \& Armstrong (1997), Harris (1999), Jackson (1999), Board et al. (2005), Grantham et al. (2006) and G. H. Grantham \& R. A. Armstrong (unpubl. data).

In addition, equivalents of the megacrystic granitic augen gneiss Culicui Suite and heterogeneous medium-grained, equigranular tonalitic orthogneiss Mocuba Suite, both volumetrically significant lithological units in the Nampula Block, are recognized in western DML in the form of the Kirwanveggen megacrystic orthogneiss (Grantham et al. 1995) and the Kvervelknatten orthogneiss (Grantham et al. 1995, 1997; Groenewald et al. 1995; Wareham et al. 1998), respectively. The chemistries of these two units are comparable, as are the ages, which are typically $c .1070-1090$ and c. 1110-1140 Ma, respectively.

Progressing further eastwards into central DML (excluding Schirmacher Hills), the western Mühlig-Hofmannfjella has crystallization ages and metamorphic ages largely comparable with those of the Nampula and western DML areas of Antarctica (Fig. 5c and d). The data from central DML are summarized in Tables 7 and 8 , with the data sources including Jacobs et al. (1998, $2003 a-c$ ), Paulsson \& Austrheim (2003), Bisnath et al. (2006). The crystallization ages recorded for central DML are in the range of 450-650 Ma and $1050-1200 \mathrm{Ma}$ and the metamorphic ages 500-600 and 1000-1100 Ma. The 550-650 Ma crystallization ages from central DML are all collected from the extreme eastern end of central DML from charnockites and anorthosites in the Wolthaat Massif area and are not recognized in the western Mühlig-Hofman Mountains. Charnockites and anorthosites of Neoproterozoic age are not recognized in the Nampula Block.

In contrast, limited data from the granulites exposed in Schirmacher Hills in NE MühligHofmannfjella (Table 8) dominantly have ages in the range $c$. 550-700 Ma with a few older ages between 800 and $1150 \mathrm{Ma}$ being recognized (Ravikant et al. 2004, 2008). Consequently, the Schirmacher Hills has ages comparable with those of the north of the Lurio Namuno Block (Fig. 5e).

Further east in the Sør Rondane area of Antarctica, SHRIMP (Shiraishi et al. 2008) and chemical $\mathrm{Th}-\mathrm{U}$-total $\mathrm{Pb}$ isochron method (CHIME) data (Asami et al. 2005) indicate that the NE Sør Rondane has age distributions similar 
Table 3. Metamorphic ages used for the Namuno Block

\begin{tabular}{|c|c|c|c|c|c|}
\hline Rock type and sample number & Method & Age (Ma) & Error & Area & Source \\
\hline Charnockitic gneiss (MA8) & SHRIMP & 572 & 9 & S. Malawi & Johnson et al. (2005) \\
\hline Felsic granulite (MA12) & SHRIMP & 547 & 10 & S. Malawi & Johnson et al. (2005) \\
\hline $\begin{array}{l}\text { Biotite-hornblende gneiss } \\
\text { (MA13) }\end{array}$ & SHRIMP & 564 & 4 & S. Malawi & Johnson et al. (2005) \\
\hline $\begin{array}{l}\text { Hofineir Gneiss deformed } \\
\text { quartzofeldspathic gneiss }\end{array}$ & SHRIMP & 536 & 10 & S. Irumide area & Johnson et al. (2006) \\
\hline Garnet-bearing pelitic migmatite & SHRIMP & 1942 & 5 & S. Irumide area & Johnson et al. (2006) \\
\hline $\begin{array}{l}\text { Nyamadzi Gneiss deformed } \\
\text { quartzofeldspathic gneiss }\end{array}$ & SHRIMP & 1065 & 13 & S. Irumide area & Johnson et al. (2006) \\
\hline $\begin{array}{l}\text { Wutepo Gneiss deformed } \\
\text { hornblende-biotite gneiss }\end{array}$ & SHRIMP & 555 & 11 & S. Irumide area & Johnson et al. (2006) \\
\hline Titanite in mafic gneiss & SHRIMP & 549 & 7 & S. Irumide area & Grantham et al. (2007a) \\
\hline $\begin{array}{l}\text { Ocua Complex MZ05045a } \\
\text { charnockite }\end{array}$ & SHRIMP & 555 & 5 & N. of Lurio & Macey et al. (2007) \\
\hline Mugeba Complex & SHRIMP & 614 & 8 & Mugeba & Kröner et al. (2001) \\
\hline
\end{tabular}

Additional data are from Jamal (2005) and Bingen et al. (2006).

to those of the Namuno Block (Fig. 5f). Almost all of these samples are from the NE Sør Rondane. The SW Sør Rondane is separated from the NE Sør Rondane by a $c .10 \mathrm{~km}$ wide shear zone (Shiraishi et al. 1991; Shiraishi \& Kagami 1992). The crystallization ages from Sør Rondane range between 1200 and $500 \mathrm{Ma}$ with most being between 500 and $650 \mathrm{Ma}$ whereas metamorphic ages are between
500 and $650 \mathrm{Ma}$ with no Mesoproterozoic metamorphism being recognized.

\section{Sri Lanka}

Very few SHRIMP zircon $\mathrm{U} / \mathrm{Pb}$ or single-grain zircon data from single suites are available from Sri Lanka. The available data (Kröner et al. 1987,

Table 4. Metamorphic ages from the Nampula Block

\begin{tabular}{|c|c|c|c|c|c|}
\hline Unit & Rock type & Method & Age (Ma) & Error & Source \\
\hline Mugeba Complex & Granulite zircon rim & SHRIMP & 591 & 4 & Macey et al. (2007) \\
\hline Mocuba Suite & $\begin{array}{l}\text { Quartzofeldspathic gneiss } \\
\text { zircon rim }\end{array}$ & SHRIMP & 527 & 18 & Macey et al. (2007) \\
\hline Mocuba Suite & Migmatitic vein & SHRIMP & 1063 & 47 & Macey et al. (2007) \\
\hline Molocue Group & $\begin{array}{l}\text { Quartzofeldspathic gneiss } \\
\text { zircon rim }\end{array}$ & SHRIMP & 502 & 90 & Macey et al. (2007) \\
\hline Culicui Suite & Augen gneiss zircon rim & SHRIMP & 538 & 8 & Macey et al. (2007) \\
\hline Culicui Suite & Augen gneiss zircon rim & SHRIMP & 525 & 20 & Macey et al. (2007) \\
\hline Culicui Suite & Charnockite zircon rim & SHRIMP & 513 & 10 & Macey et al. (2007) \\
\hline Rapale Gneiss & $\begin{array}{l}\text { Tonalitic gneiss lower } \\
\text { intercept }\end{array}$ & SHRIMP & 449 & 95 & Macey et al. (2007) \\
\hline Culicui Suite & Augen gneiss zircon rim & SHRIMP & 505 & 10 & Macey et al. (2007) \\
\hline Mamala Fm. & $\begin{array}{l}\text { Quartzofeldspathic gneiss } \\
\text { zircon rim }\end{array}$ & SHRIMP & 555 & 12 & Macey et al. (2007) \\
\hline $\begin{array}{l}\text { Leucosome S3 in } \\
\text { Culicui Suite }\end{array}$ & Leucosome & SHRIMP & 490 & 8 & Macey et al. (2007) \\
\hline Mocuba Suite & $\begin{array}{l}\text { Tonalitic gneiss } \\
\text { zircon rim }\end{array}$ & SHRIMP & 1090 & 34 & Macey et al. (2007) \\
\hline Rapale Gneiss & $\begin{array}{l}\text { Tonalitic gneiss lower } \\
\text { intercept }\end{array}$ & SHRIMP & 608 & 42 & Macey et al. (2007) \\
\hline Monapo Complex & Granulite zircon rim & SHRIMP & 579 & 11 & Grantham et al. (2007b) \\
\hline Monapo Complex & Syenite zircon rim & SHRIMP & 596 & 5 & Grantham et al. (2007b) \\
\hline
\end{tabular}

Additional data are available from Jamal (2005). 


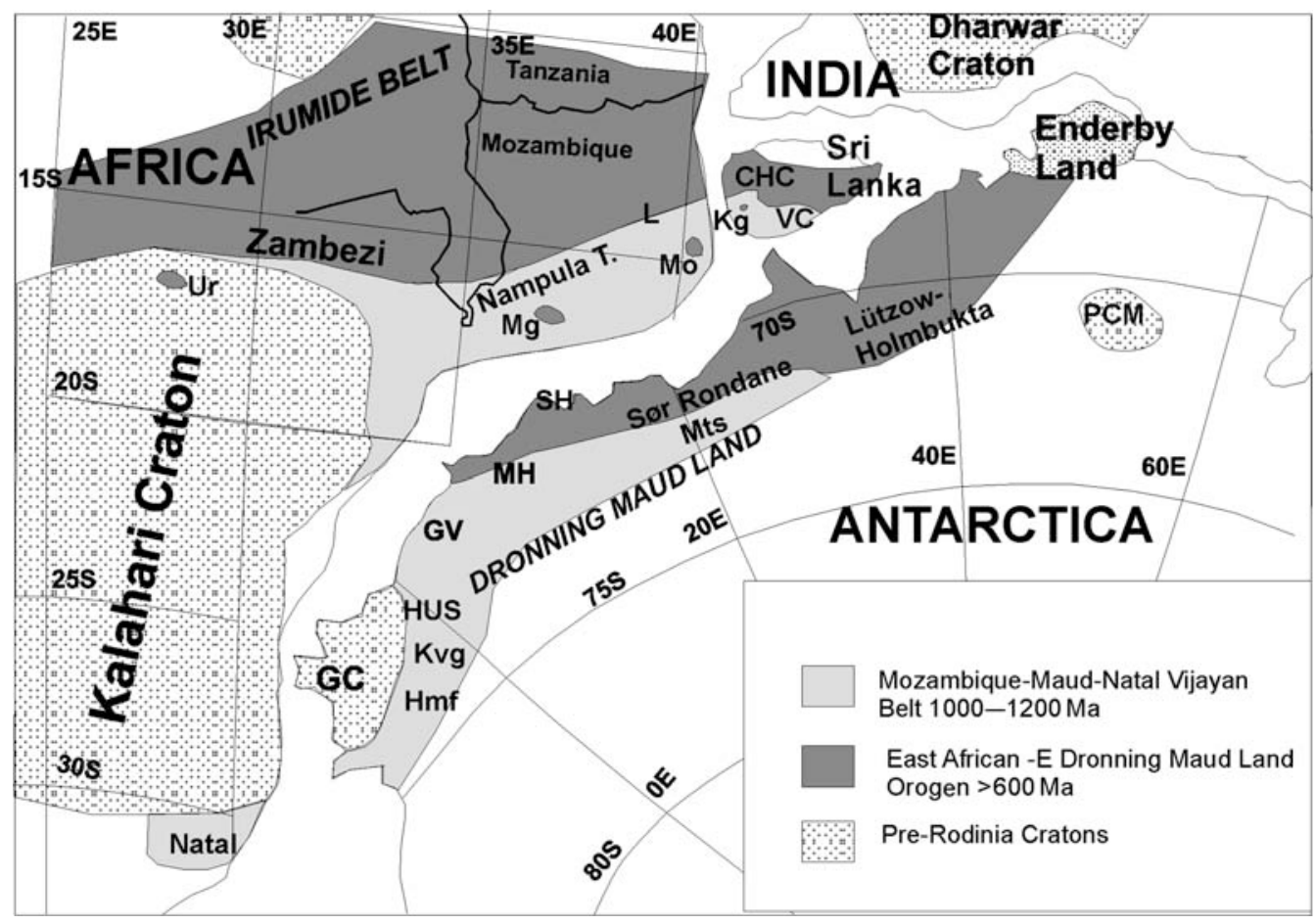

Fig. 4. Schematic map of the various crustal blocks belonging to the Namuno and Nampula-type blocks. VC, Vijayan Complex; CHC, Central Highland Complex; Mo, Monapo Klippen; Mg, Mugeba Klippen; Kg, Kataragama Klippen; L, Lurio Belt; SH, Schirmacher Hills; MH, Mühlig-Hofmannfjella; GV, Gjelsvikfjella; HUS, Sverdrupfjella; Kvg, Kirwanveggen; Hmf, Heimefrontfjella; GC, Grunehogna Craton; Ur, Urungwe Klippen; PCM, Prince Charles Mountain.

1994; Baur et al. 1991; Holzl et al. 1994) are largely derived from multigrain TIMS studies on single rock units or come from SHRIMP studies on metasediments aimed at provenance determinations. None the less, histograms summarizing data from the Highland Complex (HC) (Fig. 5g) and Vijayan Complex (VC) (Fig. 5h) of Sri Lanka show that the available data from the $\mathrm{HC}$ have a similar pattern to those for the Namuno Block of Mozambique and the Sør Rondane and Schirmacher Hills areas of Antarctica, whereas the VC has a pattern of ages comparable to those of the Nampula Block and DML, Antarctica. The data reported from the Vijayan Complex by Kröner et al. (1987) have large analytical uncertainties, resulting in the broad curves defined by the probability density distribution (Fig. 5h), whereas the absolute ages show age ranges of 500-600 and 1000$1250 \mathrm{Ma}$.

In conclusion, the chronological data combined with lithological varieties facilitate recognition of two age groups of tectonic blocks; namely, those with significant volumes of rock with ages between 600 and $900 \mathrm{Ma}$ and those without (Fig. 4). The former group comprises the north of the Lurio Namuno Block, Malawi and southern Irumide Block as well as the Mugeba and Monapo klippen, the Highland Complex in Sri Lanka, the NE Sør Rondane, the far eastern Mühlig-Hofmannfjella and the Schirmacher Hills. The latter group comprises the Nampula Block, the Vijayan Complex in Sri Lanka, the SW Sør Rondane, the western Mühlig-Hofmannfjella, Sverdrupfjella and its extensions into Kirwanveggen (Fig. 4). In the remainder of the paper, we will refer to these grouped blocks as the Namuno and Nampula Blocks, respectively.

\section{Structural data}

In most cases the boundaries between the Namuno and Nampula Blocks (when exposed) are defined by highly sheared rocks. In NE Mozambique the boundary is represented by the highly sheared Lurio Belt and the circumferential mylonites 

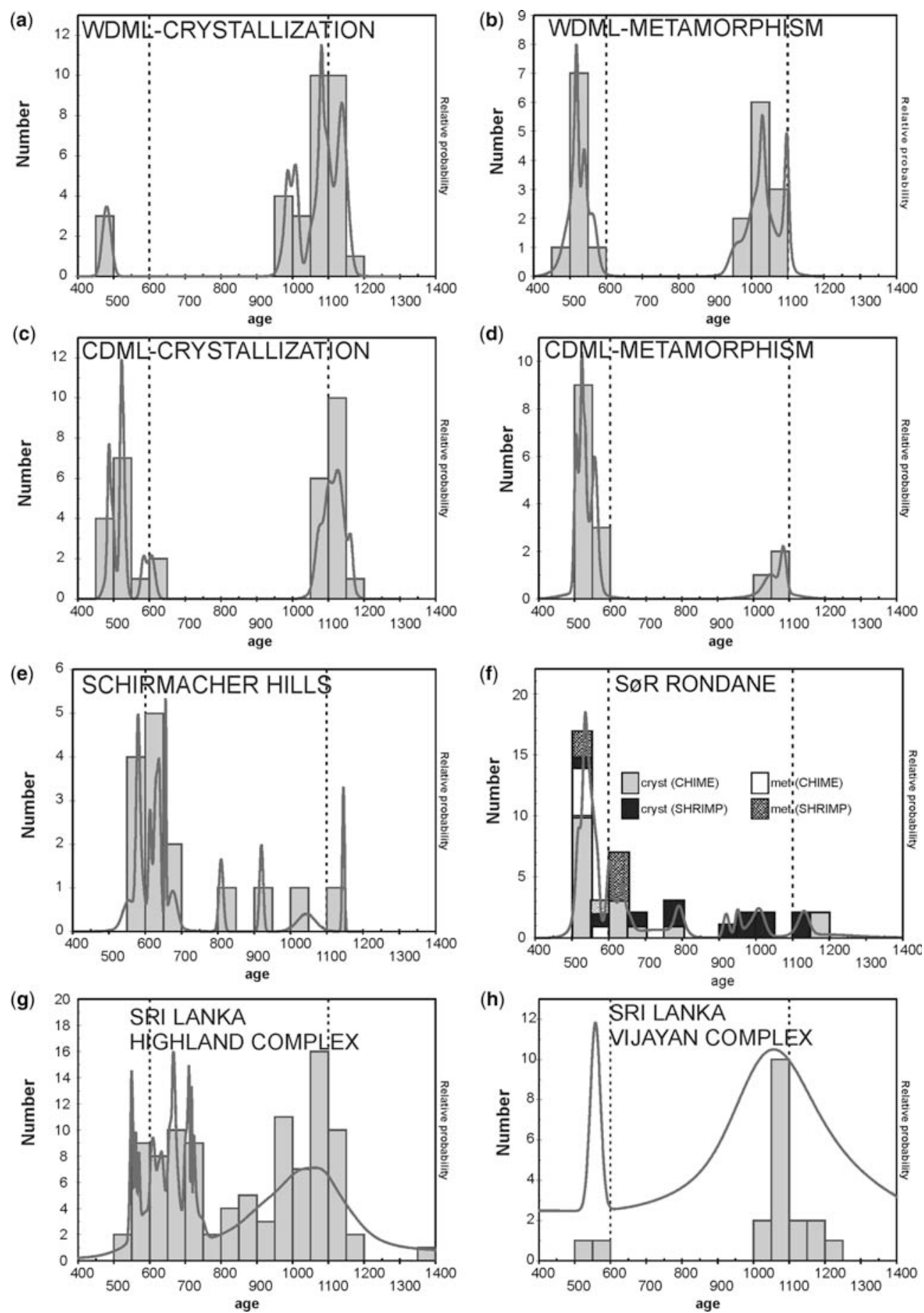

Fig. 5. Histograms of geochronological data from western DML (a, b), central DML (c, d), Schirmacher Hills (e), Sør Rondane (f), Sri Lanka (Highland Complex) (g) and Sri Lanka (Vijayan Complex) (h). The $600 \mathrm{Ma}$ and $1100 \mathrm{Ma}$ lines are shown for reference. The data from Sør Rondane are subdivided into SHRIMP-based ages of crystallization and metamorphism and CHIME-based ages of crystallization and metamorphism. 
Table 5. Crystallization ages from Sverdrupfjella and Kirwanveggen, western Dronning Maud Land

\begin{tabular}{|c|c|c|c|c|}
\hline Unit & Method & Age (Ma) & Error & Source \\
\hline Kyanite leucogneiss & SHRIMP & 1096 & 10 & Harris (1999) \\
\hline $\mathrm{Bt}$-grt migmatite & SHRIMP & 1157 & 10 & Harris (1999) \\
\hline Intrusive leucogneiss & SHRIMP & 1101 & 13 & Harris (1999) \\
\hline Megacrystic orthogneiss & SHRIMP & 1088 & 10 & Harris (1999) \\
\hline Pegmatite vein & SHRIMP & 1079 & 6 & Harris (1999) \\
\hline Late felsic dyke & SHRIMP & 1011 & 8 & Moyes \& Harris (1996) \\
\hline Sveabreen Granite & SHRIMP & 1127 & 12 & Moyes \& Harris (1996) \\
\hline Fugitive Granite & SHRIMP & 1131 & 25 & Moyes \& Harris (1996) \\
\hline Fugitive Granite & SHRIMP & 1061 & 14 & Moyes \& Harris (1996) \\
\hline Roerkulten Granite & SHRIMP & 1103 & 13 & Moyes \& Harris (1996) \\
\hline Rootshorga Paragneiss & SHRIMP & 1092 & 13 & Moyes \& Harris (1996) \\
\hline Granite gneiss tonalitic Wbsv065 & SHRIMP & 1132 & 16 & Board et al. (2005) \\
\hline Tabular granite Wbsv073 & SHRIMP & 1072 & 10 & Board et al. (2005) \\
\hline Granite dykes Wbsv069 & SHRIMP & 480 & 10 & Board et al. (2005) \\
\hline Kvervelkatten Gneiss & SHRIMP & 1134 & 11 & Jackson (1999) \\
\hline Kvervelkatten Amphibolite Pod Cjk151 & SHRIMP & 1139 & 10 & Jackson (1999) \\
\hline Megacrystic augen gneiss Cjk158 & SHRIMP & 1074 & 11 & Jackson (1999) \\
\hline Leucopegmatite Cjk155 & SHRIMP & 1050 & 10 & Jackson (1999) \\
\hline Porphyritic granite dyke Cjk 103 & SHRIMP & 1011 & 8 & Jackson (1999) \\
\hline Leucogranite Cjk152 & SHRIMP & 990 & 12 & Jackson (1999) \\
\hline Granite dyke Cjk 159 & SHRIMP & 980 & 13 & Jackson (1999) \\
\hline Amphibolite dyke Hallgrens Cjk160 & SHRIMP & 986 & 6 & Jackson (1999) \\
\hline Banded bt gneiss Hallgrens Cjk56 & SHRIMP & 1081 & 4 & Jackson (1999) \\
\hline Banded bt gneiss Hallgrens Cjk59 & SHRIMP & 994 & 22 & Jackson (1999) \\
\hline Titanite Cjk 159 & SHRIMP & 1003 & 9 & Jackson (1999) \\
\hline Grey gneiss & SHRIMP & 1143 & 11 & Jackson (1999) \\
\hline Dalmatian Granite & SHRIMP & 489 & 10 & Krynauw \& Jackson (1996) \\
\hline Brattskarvet Monzonite & SHRIMP & 474 & 10 & Krynauw \& Jackson (1996) \\
\hline Midbressrabben Diorite & SHRIMP & 1140 & 10 & $\begin{array}{l}\text { G. H. Grantham \& R. A. } \\
\text { Armstrong (unpubl. data) }\end{array}$ \\
\hline Grey gneiss Jutulrora Jw4 & SHRIMP & 1139 & 12 & $\begin{array}{l}\text { G. H. Grantham \& R. A. } \\
\text { Armstrong (unpubl. data) }\end{array}$ \\
\hline Augen gneiss Sa 10 & SHRIMP & 1096 & 14 & $\begin{array}{l}\text { G. H. Grantham \& R. A. } \\
\text { Armstrong (unpubl. data) }\end{array}$ \\
\hline
\end{tabular}

around the Monapo and Mugeba klippen. In the west the Sanangoe thrust zone (Barr \& Brown 1987) and the shear zones defining the allochthonous Tete Complex (Koistinen et al. 2006; Westerhof 2006) represent possible extensions of the Lurio Belt.

In Sri Lanka, the boundary between the Highland Complex is interpreted as a complex thrustfault zone (Kleinschrodt 1994) in which the granulite-facies Highland Complex has been thrust-faulted over the amphibolite-facies Vijayan Complex. In the shear zone, which is reportedly hundreds of metres wide, a strong north-south-oriented stretching lineation is developed; however, kinematic indicators are sparse.

In Sør Rondane, NE Sør Rondane is separated from SW Sør Rondane by a $c .10 \mathrm{~km}$ wide shear zone (Shiraishi et al. 1991). The boundary in Mühlig-Hofmannfjella is, however, not exposed. The differences in reported geochronology and lithologies in eastern Mühlig-Hofmannfjella imply that the boundary between the two blocks probably passes immediately east of the Wolthaat Anorthosite Massif, the most easterly nunatak group in Mühlig-Hofmannfjella, whose age and extensional affinity suggest that it belongs to the Namuno Block along with the granulites at Schirmacher Hills and Mramornye nunataks. This is possibly also supported by the differences in structural histories between the Wolthaat Massif and rocks further east described by Bauer et al. (2004). Immediately east of the Wolthaat Massif, the nunataks are reportedly underlain by $c$. $550 \mathrm{Ma}$ granites after which, progressing eastwards, the lithologies are typical of the Nampula Block (Jacobs et al. 2003c; Bauer et al. 2004). The lithologies astride the Orvinfjella shear zone in Mühlig-Hofmannfjella reportedly are not different (Jacobs et al. 2003c; Bauer et al. 2004) and the orientation of the Orvinfjella Shear Zone suggests that it may 
Table 6. Metamorphic ages from Sverdrupfjella and Kirwanveggen, western Dronning Maud Land

\begin{tabular}{|c|c|c|c|c|}
\hline Unit & Method & Age (Ma) & Error & Source \\
\hline Leucosome in garnet migmatite gneiss & SHRIMP & 1098 & 5 & Harris (1999) \\
\hline Kyanite leucogneiss & SHRIMP & 1096 & 10 & Harris (1999) \\
\hline Granite gneiss rim tonalitic wbsv065 & SHRIMP & 1031 & 47 & Board et al. (2005) \\
\hline Metapelite wbsv025 & SHRIMP & 1044 & 47 & Board et al. (2005) \\
\hline Metapelite wbsv025 & SHRIMP & 540 & 6 & Board et al. (2005) \\
\hline Tabular granite rim wbsv073 & SHRIMP & 565 & 11 & Board et al. (2005) \\
\hline Tabular granite rim wbsv073 & SHRIMP & 996 & 17 & Board et al. (2005) \\
\hline Leucosome wbsv071 & SHRIMP & 1035 & 31 & Board et al. (2005) \\
\hline Leucosome wbsv071 lower intercept & SHRIMP & 499 & 17 & Board et al. (2005) \\
\hline Leucosome wbsv074 & SHRIMP & 515 & 7 & Board et al. (2005) \\
\hline Leucosome wbsv 113 & SHRIMP & 1032 & 15 & Board et al. (2005) \\
\hline Leucosome wbsv113 & SHRIMP & 503 & 35 & Board et al. (2005) \\
\hline Leucosome wbsv 114 & SHRIMP & 525 & 35 & Board et al. (2005) \\
\hline Leucosome wbsv116 & SHRIMP & 519 & 4 & Board et al. (2005) \\
\hline Rim Kvervelknatten & SHRIMP & 1060 & 22 & $\begin{array}{l}\text { Krynauw \& Jackson } \\
\text { (1996) }\end{array}$ \\
\hline Leucosome CJK153 & SHRIMP & 1031 & 6 & Jackson (1999) \\
\hline Monazite CJK 149 & SHRIMP & 956 & 17 & Jackson (1999) \\
\hline Titanite CJK56 & SHRIMP & 1015 & 16 & Jackson (1999) \\
\hline Rim SA10 & SHRIMP & 538 & 25 & $\begin{array}{l}\text { G. H. Grantham \& } \\
\text { R. A. Armstrong } \\
\text { (unpubl. Data) }\end{array}$ \\
\hline Mafic dyke RK55 & SHRIMP & 523 & 21 & Grantham et al. (2006) \\
\hline
\end{tabular}

continue into Mozambique as the Namama ShearZone (Grantham et al. 2003), where it dissects similar rock types. Piazolo (2004) has described the structural evolution of the Mramornye nunataks and Schirmacher Hills. Both areas are characterized by strong shear fabrics, with those at Mramornye nunataks suggesting thrust-faulting toward the south during $\mathrm{D}_{3}$.

\section{Planar fabric data}

Grantham et al. (2003) described one of the enigmas of the correlation of northern Mozambique with Dronning Maud Land as being the opposing structural facing directions in the two areas. Planar structures in northern Mozambique were described as dipping dominantly to the north and NW whereas those in western Dronning Maud Land were described as dipping dominantly to the SE. The improved densities of structural observation and geochronology permit the conclusion that the ages of fabrics in the two areas are not the same.

The strong planar NW-dipping fabrics in and adjacent to the Lurio Belt clearly affect rocks with crystallization ages of $c$. $630 \mathrm{Ma}$ along with older rocks in the Nampula Block. Consequently, the strong fabric-producing event in these areas has to be younger than $c$. $630 \mathrm{Ma}$. The structural data from the Mugeba and Monapo klippen, particularly the latter, are discordant to their structurally underlying rocks, supporting their interpretation as klippen (Grantham et al. 2007b; Macey et al. 2007). The Monapo Complex, in particular, is interpreted as a circular synformal remnant in which at least two phases of deformation defined by the layered granulites form a large type 2 interference fold structure (Grantham et al. 2007b).

In contrast to the younger than $630 \mathrm{Ma}$ deformation in the klippen and the Lurio Belt, a detailed study in southern Kirwanveggen by Jackson (1999) showed that most of the deformation occurred there before $c .900 \mathrm{Ma}$. This is circumstantially supported in southern Kirwanveggen at Skappelknabben, where strongly sheared augen gneisses with greenschist-grade, planar fabrics are in relative close proximity to the virtually undeformed (brittlefaulted) sandstones of the Urfjell Group at Drapane. The Urfjell Group is less than c. 550 Ma old (Moyes et al. 1997), the age of the youngest detrital zircon recorded in it (Croaker 1999). Grantham et al. (2006) have shown that a c. $950 \mathrm{Ma}$ mafic dyke at Roerkulten in Sverdrupfjella post-dates an earlier migmatitic fabric-forming event $\left(\mathrm{D}_{1}\right)$, has a planar fabric $\left(D_{2}\right)$ that has been deformed about $D_{3}$ folds, and was metamorphosed in the upper amphibolite facies at c. $500 \mathrm{Ma}$. Similarly, the syntectonic emplacement of granitic sheets at c. $480 \mathrm{Ma}$ during top-to-the-SE-directed deformation in NW Sverdrupfjella (Grantham et al. 1991) demonstrates 
Table 7. Crystallization ages for Mühlig-Hofmannfjella in central Dronning Maud Land

\begin{tabular}{|c|c|c|c|c|}
\hline Rock unit or type and sample number & Method & Age (Ma) & Error & Source \\
\hline Stabben gabbro & SHRIMP & 487 & 4 & Bisnath et al. (2006) \\
\hline Granite aplite dykes & SHRIMP & 497 & 5 & Bisnath et al. (2006) \\
\hline Augen gneiss & SHRIMP & 1104 & 8 & Bisnath et al. (2006) \\
\hline Grey migmatite augen gneiss & SHRIMP & 1124 & 11 & Bisnath et al. (2006) \\
\hline Granite gneiss & SHRIMP & 1133 & 16 & Bisnath et al. (2006) \\
\hline Banded gneiss & SHRIMP & 1091 & 16 & Bisnath et al. (2006) \\
\hline Granite gneiss & SHRIMP & 1130 & 19 & Bisnath et al. (2006) \\
\hline Homogeneous migmatite & SIMS & 1163 & 6 & Paulsson \& Austrheim (2003) \\
\hline Stabben syenite & SIMS & 500 & 8 & Paulsson \& Austrheim (2003) \\
\hline Lamprophyre dyke Risemedet/2312/2 & SHRIMP & 523 & 5 & Jacobs et al. (2003a) \\
\hline Charnockite Hochlinfjellet $1301 / 2$ & SHRIMP & 521 & 3 & Jacobs et al. $(2003 a)$ \\
\hline Augen gneiss $2412 / 4$ & SHRIMP & 1096 & 8 & Jacobs et al. $(2003 b)$ \\
\hline Grey migm gneiss $2712 / 4$ & SHRIMP & 1115 & 12 & Jacobs et al. $(2003 b)$ \\
\hline Augen gneiss $1512 / 1$ & SHRIMP & 1123 & 21 & Jacobs et al. $(2003 b)$ \\
\hline Grey gneiss $1701 / 2$ & SHRIMP & 1142 & 21 & Jacobs et al. $(2003 b)$ \\
\hline Migmatitic augen gneiss & SHRIMP & 1137 & 14 & Jacobs et al. $(2003 b)$ \\
\hline Stabben gabbro & SHRIMP & 483 & 11 & Jacobs et al. $(2003 a)$ \\
\hline Granite dyke Gygra & SHRIMP & 487 & 4 & Jacobs et al. $(2003 a)$ \\
\hline Felsic gneiss j1671 & SHRIMP & 1073 & 9 & Jacobs et al. (1998) \\
\hline Felsic gneiss j1704 & SHRIMP & 1137 & 21 & Jacobs et al. (1998) \\
\hline Felsic gneiss j1795 & SHRIMP & 1076 & 14 & Jacobs et al. (1998) \\
\hline Orthogneiss j1736 & SHRIMP & 1086 & 20 & Jacobs et al. (1998) \\
\hline Orthogneiss j1797 & SHRIMP & 1087 & 28 & Jacobs et al. (1998) \\
\hline Metagranodiorite j1698 & SHRIMP & 530 & 8 & Jacobs et al. (1998) \\
\hline Metaleucogranite j1695 & SHRIMP & 527 & 6 & Jacobs et al. (1998) \\
\hline Felsic gneiss j1838 & SHRIMP & 1130 & 12 & Jacobs et al. (1998) \\
\hline Charnockite j1886 & SHRIMP & 608 & 9 & Jacobs et al. (1998) \\
\hline Anorthosite j1955 & SHRIMP & 600 & 12 & Jacobs et al. (1998) \\
\hline Anorthosite j1958 & SHRIMP & 583 & 7 & Jacobs et al. (1998) \\
\hline Zwiesel gabbro & SHRIMP & 527 & 5 & Jacobs et al. $(2003 c)$ \\
\hline Zwiesel gabbro & SHRIMP & 521 & 6 & Jacobs et al. $(2003 c)$ \\
\hline
\end{tabular}

a direction of deformation similar to that in Mozambique. These data support the work of Grantham et al. (1995), who concluded that there were two major periods of deformation in Sverdrupfjella in western DML; namely, one during the Mesoproterozoic at $c .900-1000 \mathrm{Ma}$ and the other during the Neoproterozoic and into the Cambrian at $c$. 550-490 Ma. The data do not support the suggestion by Board et al. (2005) that the dominant deformation in Sverdrupfjella is Neoproterozoic to Cambrian in age.

A more detailed study of planar structures (Figs 6-8) in the various areas of Mozambique and DML, Antarctica provides a better understanding of the structural relationships.

In northern Mozambique, planar fabrics (Fig. 6a and b) dip and plunge dominantly toward the north to NW in the Lurio Belt as well as in the area along the southern margin of the Lurio Belt. Similarly the lineations in the same areas (Fig. 6e) define an arc with lineations plunging between north and west with concentrations towards the north and WNW. Limited fold-axis data are similar to the lineations shown in Figure 6e. Progressing southwards toward the northern Mozambique coast the structural patterns become more complex and bimodal in nature, with both north- to NW-dipping planar structures as well as south- to SE-dipping structures being common (Fig. 6c and d). Similarly, the lineations along the southern margin of the northern Mozambique coast also show a bimodal variation with west- and ESE-dipping orientations (Fig. 6f). The data in Figure $5 \mathrm{f}$ are skewed by the high number of readings collected in the broad vicinity of the Namama shear zone by Aquater in the early 1980s (Aquater 1983).

Bimodal patterns in the planar fabrics are observed in the western Mühlig-Hofman Mountains of Antarctica (Fig. 7f; data from Jacobs et al. 2003a) and the Gjelsvikfjella (Fig. 7g, A. Bisnath unpubl. data), with gneisses dipping broadly to the $\mathrm{NE}$ and SW. In contrast, the structural data from Mramornye nunataks (Fig. 7h; from Piazolo 2004) is unimodal, with the gneisses dipping dominantly shallowly to steeply toward the ENE. Lineations from Gjelsvikfjella (Fig. 7a) show at least three 
Table 8. Metamorphic ages from Mühlig-Hofmannfjella and Schirmacher Hills

\begin{tabular}{|c|c|c|c|c|}
\hline Subject or sample and sample number & Method & Age (Ma) & Error & Source \\
\hline Age of migmatization & SHRIMP & 504 & 4 & Paulsson \& Austrheim (2003) \\
\hline Zircon overgrowths & SIMS & 504 & 6 & Paulsson \& Austrheim (2003) \\
\hline Migmatite gneiss & SHRIMP & 529 & 4 & Bisnath et al. (2006) \\
\hline Lower intercept $\mathrm{Aba} / 32$ & SHRIMP & 527 & 50 & Bisnath et al. (2006) \\
\hline Leucosome-1301/2 & SHRIMP & 521 & 3 & Jacobs et al. (2003a) \\
\hline Leucosome-0801/3 & SHRIMP & 558 & 6 & Jacobs et al. $(2003 a)$ \\
\hline Grey gneiss $1701 / 2$ & SHRIMP & 1061 & 56 & Jacobs et al. $(2003 b)$ \\
\hline Grey gneiss $1701 / 2$ & SHRIMP & 528 & 10 & Jacobs et al. $(2003 b)$ \\
\hline Augen gneiss $1512 / 1$ & SHRIMP & 1049 & 19 & Jacobs et al. $(2003 b)$ \\
\hline Leucosome & SHRIMP & 516 & 5 & Jacobs et al. (1998) \\
\hline Rim j1704 & SHRIMP & 522 & 10 & Jacobs et al. (1998) \\
\hline $\operatorname{Rim}$ j1795 & SHRIMP & 557 & 11 & Jacobs et al. (1998) \\
\hline J1886 charnockite & SHRIMP & 544 & 15 & Jacobs et al. (1998) \\
\hline Anorthosite rim j1955 & SHRIMP & 555 & 11 & Jacobs et al. (1998) \\
\hline Metamorphic rim j1704 & SHRIMP & 1084 & 8 & Jacobs et al. (1998) \\
\hline Whole-rock minerals & TIMS Sm-Nd & 616 & 52 & Ravikant et al. (2004) \\
\hline Whole-rock minerals & TIMS Sm-Nd & 632 & 8 & Ravikant et al. (2004) \\
\hline Whole-rock minerals & TIMS Sm-Nd & 554 & 16 & Ravikant et al. (2004) \\
\hline Monazite & TIMS U/Pb & 629 & 5 & Ravikant et al. (2008) \\
\hline Monazite & TIMS U/Pb & 639 & 4 & Ravikant et al. (2008) \\
\hline Titanite & TIMS U/Pb & 580 & 5 & Ravikant et al. (2008) \\
\hline Monazite & TIMS U/Pb & 809 & 6 & Ravikant et al. (2008) \\
\hline Monazite & TIMS U/Pb & 656 & 2 & Ravikant et al. (2008) \\
\hline Monazite & TIMS U/Pb & 676 & 12 & Ravikant et al. (2008) \\
\hline Monazite & TIMS U/Pb & 580 & 5 & Ravikant et al. (2008) \\
\hline Monazite & TIMS U/Pb & 613 & 4 & Ravikant et al. (2008) \\
\hline Monazite & TIMS U/Pb & 1044 & 25 & Ravikant et al. (2008) \\
\hline Monazite & TIMS U/Pb & 920 & 5 & Ravikant et al. (2008) \\
\hline Titanite & TIMS U/Pb & 589 & 9 & Ravikant et al. (2008) \\
\hline Titanite & TIMS U/Pb & 1146 & 3 & Ravikant et al. (2008) \\
\hline
\end{tabular}

significant concentrations toward the NE, SE and NW. This variation either suggests numerous shearrelated lineation-producing events or reflects the folding of earlier unimodal lineations to produce multiple directions.

Grantham et al. (1995) recorded bimodal planar structure dipping patterns in NW Sverdrupfjella as well as northern Kirwanveggen (Fig. 7a and b). In contrast, planar fabrics (Fig. 7c and d) in southeastern Sverdrupfjella and southern Kirwanveggen are unimodal and dip dominantly toward the SE (Grantham et al. 1995). Similarly, lineations in northern Sverdrupfjella (Fig. 8b) plunge dominantly eastwards with a subordinate roughly north-plunging group. In northern Kirwanveggen lineations plunge dominantly roughly north and south (Fig. 8c). In southeastern Sverdrupfjella a unimodal lineation direction is recognized plunging dominantly toward the SE (Fig. 8d).

The data suggest a zone along the southern coast of northern Mozambique and along the northern coast of DML in which bimodal structural patterns are seen in planar and linear structures. In NW
Sverdrupfjella it is apparent in the field from the wonderful 3D exposures available in Antarctica that the bimodal pattern arises from the refolding of earlier $\mathrm{D}_{1}$ and $\mathrm{D}_{2}$ SE-dipping planar fabrics about near-horizontal NE-oriented $\mathrm{D}_{3}$ fold axes (Fig. 9a and b). The $\mathrm{D}_{3}$ folds are relatively open and commonly verge toward the SE or have top-to-the-SE geometries. In contrast, the $\mathrm{D}_{1}$ and $\mathrm{D}_{2}$ folds are tight to isoclinal and commonly verge toward the NW (Fig. 9a). The folded dyke at Roerkulten reported by Grantham et al. (2006) is also typical of a $\mathrm{D}_{3}$ fold. Other examples of $\mathrm{D}_{3}$ folds with NW-dipping axial planes are seen at the southern end of Brekkerista and the western end of Roerkulten (Grantham 1992).

\section{Metamorphic history}

In Mozambique the mineral assemblages and grades of metamorphism also show significant differences. Except for the Mugeba and Monapo klippen, the grade of metamorphism in the Nampula Block 


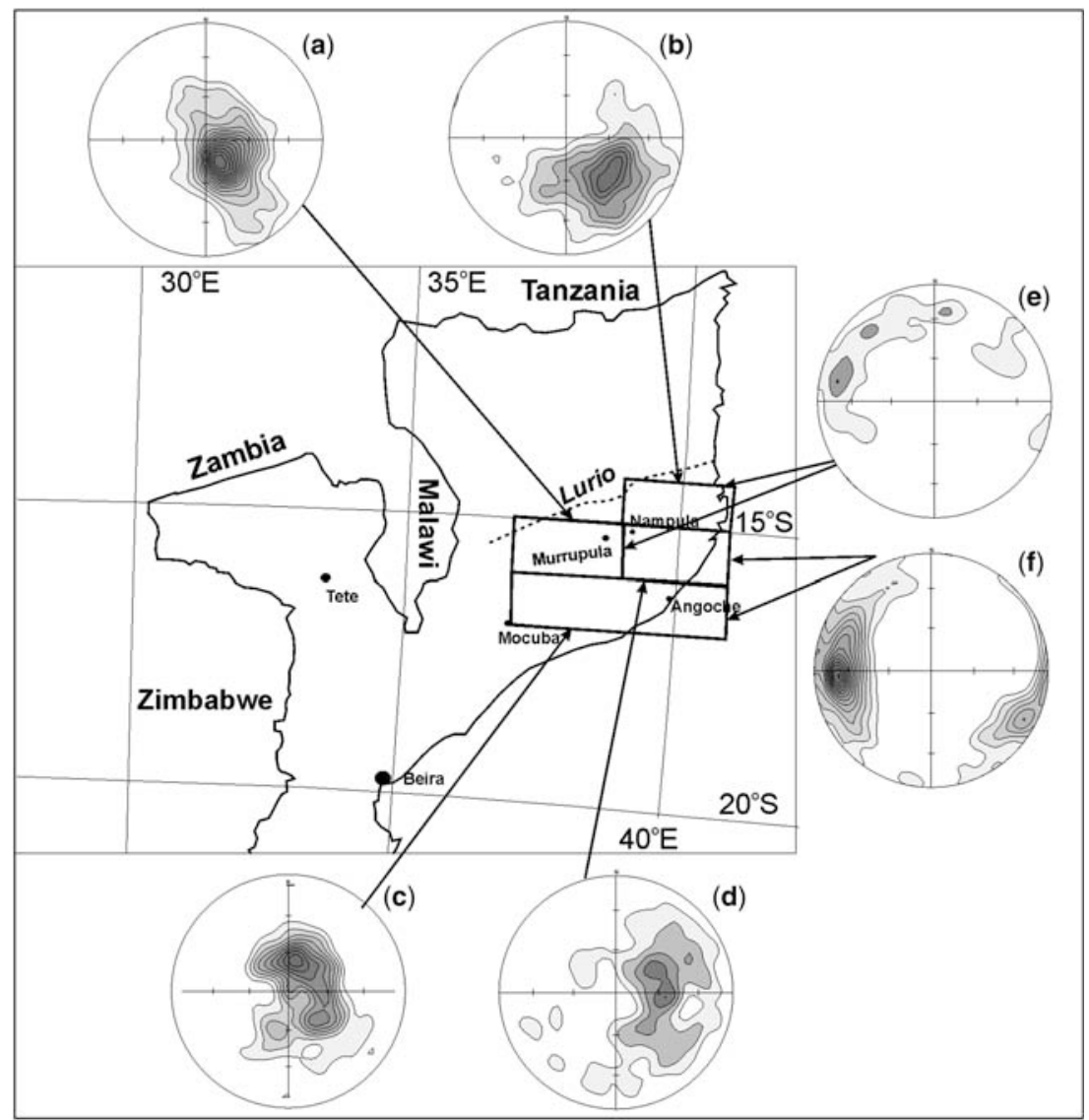

Fig. 6. Contoured stereographic projections of poles to planar structures $(\mathbf{a}-\mathbf{d})$ and stereographic projections of contoured lineations $(\mathbf{e}, \mathbf{f})$ from various areas mapped in northern Mozambique. The approximate position of the Lurio Belt is shown. The arrows connecting the stereonets to the map area borders show the approximate areas from which the data have been collected.

is typically upper amphibolite facies, with orthopyroxene being seen only rarely as relict grains in magmatic charnockite granitoids or as rare localized diffuse fluid-driven(?) vein charnockitization. The Mocuba Suite gneiss is extensively migmatized, locally showing both Mesoproterozoic and the Pan-African generations of migmatization. The post-Mocuba Suite gneisses preserve only the weakly developed Pan-African migmatization. A significant aspect of the Nampula Block, however, is the abundance of undeformed to weakly deformed granitoids and pegmatites whose ages vary between c. 495 and 530 Ma. Absolute pressure constraints are difficult to constrain for the Nampula Block because of the absence of rock types (meta-pelites, metabasites) with suitable mineral assemblages. The only reliable constraints are provided by sillimanite-bearing migmatitic quartzofeldspathic gneisses implying temperatures of at least $c .700{ }^{\circ} \mathrm{C}$ and pressures $<c$. $7-8$ kbar. Consequently, no $P-T$ path for the Nampula Block is presented here. However, the weak migmatization and development of granitic melts with ages of c. 495-530 Ma imply an increase in temperature probably to $\geq 650-750{ }^{\circ} \mathrm{C}$ during this time period. Implicit in the temperature increase is an increase in depth, because no widespread extensive source of advective heat is recognized. The granitoids are concentrated in the Nampula Block, with only a limited number of small granitoids being recognized north of the Lurio Belt.

In contrast, the rocks in the Mugeba and Monapo klippen contain granulite-grade orthogneisses and paragneisses. The orthogneisses are typically ultramafic, mafic to felsic in composition. The ultramafic rocks in the Monapo klippen comprise clinopyroxenites with $<c$. $5 \%$ modal plagioclase but c. $20 \%$ normative plagioclase, implying a 


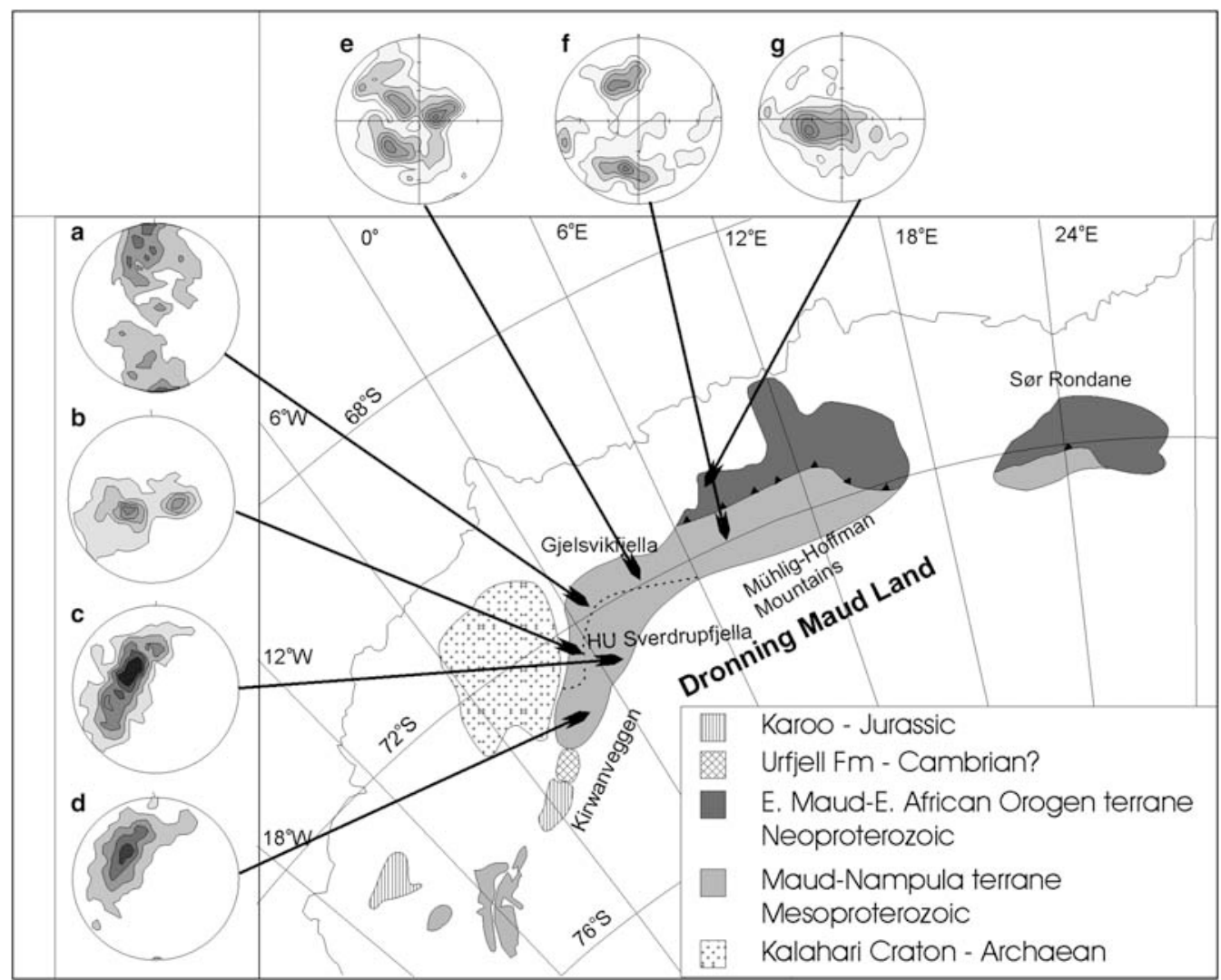

Fig. 7. Contoured stereographic projections of poles to planar structures from various localities in western and central DML.

substantial omphacitic component. Subtle vermicular intergrowths of $\mathrm{Pl}+\mathrm{Cpx}\left(c .5 \% \mathrm{Al}_{2} \mathrm{O}_{3}\right.$ ) (mineral abbreviations after Kretz 1983) are locally developed at the margins of coarse cpx grains with c. $10 \%$ $\mathrm{Al}_{2} \mathrm{O}_{3}$. These are interpreted as decompression exsolution intergrowths (G. H. Grantham, unpubl. data). The mafic rocks contain $\mathrm{Pl}-\mathrm{Opx}-\mathrm{Cpx}-\mathrm{Grt}$, and some samples have decompression textures defined by garnet with vermicular rims of $\mathrm{Cpx} / \mathrm{Hbl}+\mathrm{Pl}$. In the felsic granulites idiomorphic post-tectonic garnet (+ Qtz) after Opx/Cpx define isobaric cooling reactions. The metapelites contain $\mathrm{Grt}+\mathrm{Sill}+\mathrm{Pl}+$ Rt assemblages. Thermobarometry on these assemblages from Mugeba (Roberts et al. 2005) and Monapo (Grantham et al. 2007b) have facilitated the construction of $P-T$ loops (Fig. 10b and c).

The $P-T$ loops from Mugeba and Monapo have initial isothermal decompression from c. 900 to $1000{ }^{\circ} \mathrm{C}$ and $>c .10 \mathrm{kbar}$, followed by isobaric cooling at $c .700{ }^{\circ} \mathrm{C}$ and $c .6-7 \mathrm{kbar}$. These $P-T$ loops are comparable with those from Schirmacher Hills (Fig. 10e), Sør Rondane (Fig. 10d) and the
Highland Complex of Sri Lanka (Fig. 10a). All these $P-T$ loops suggest early isothermal decompression followed by isobaric cooling with $P-T$ conditions of $c .6-7 \mathrm{kbar}$ and $c .600-700{ }^{\circ} \mathrm{C}$ at c. $550 \mathrm{Ma}$, although the $P-T$ path from $\mathrm{S} ø \mathrm{r}$ Rondane shows additional complexities not recognized from other areas. The $P-T$ conditions for Schirmacher Hills and Sør Rondane are from Baba et al. (2006) whereas those from Sri Lanka are from Schumacher et al. (1990), Hiroi et al. (1994) and Raase \& Schenk (1994).

The $P-T$ conditions in Gjelsvikfjella are from Bisnath \& Frimmel (2005; Fig. 10f) and show an isothermal decompression path from c. $10 \mathrm{kbar}$ and $700-800{ }^{\circ} \mathrm{C}$ during the Mesoproterozoic toward c. $5 \mathrm{kbar}$ and $c .650^{\circ} \mathrm{C}$ at $550 \mathrm{Ma}$. Extensive granitoids of $c$. $550 \mathrm{Ma}$ age are recognized in Gjelsvikfjella and Mühlig-Hofmannfjella as in the Nampula terrane, also implying a thermal increase at this time. The $P-T$ path described by Grantham et al. (1995) (Fig. 10g) is similar to that described for Gjelsvikfjella and is also characterized by 


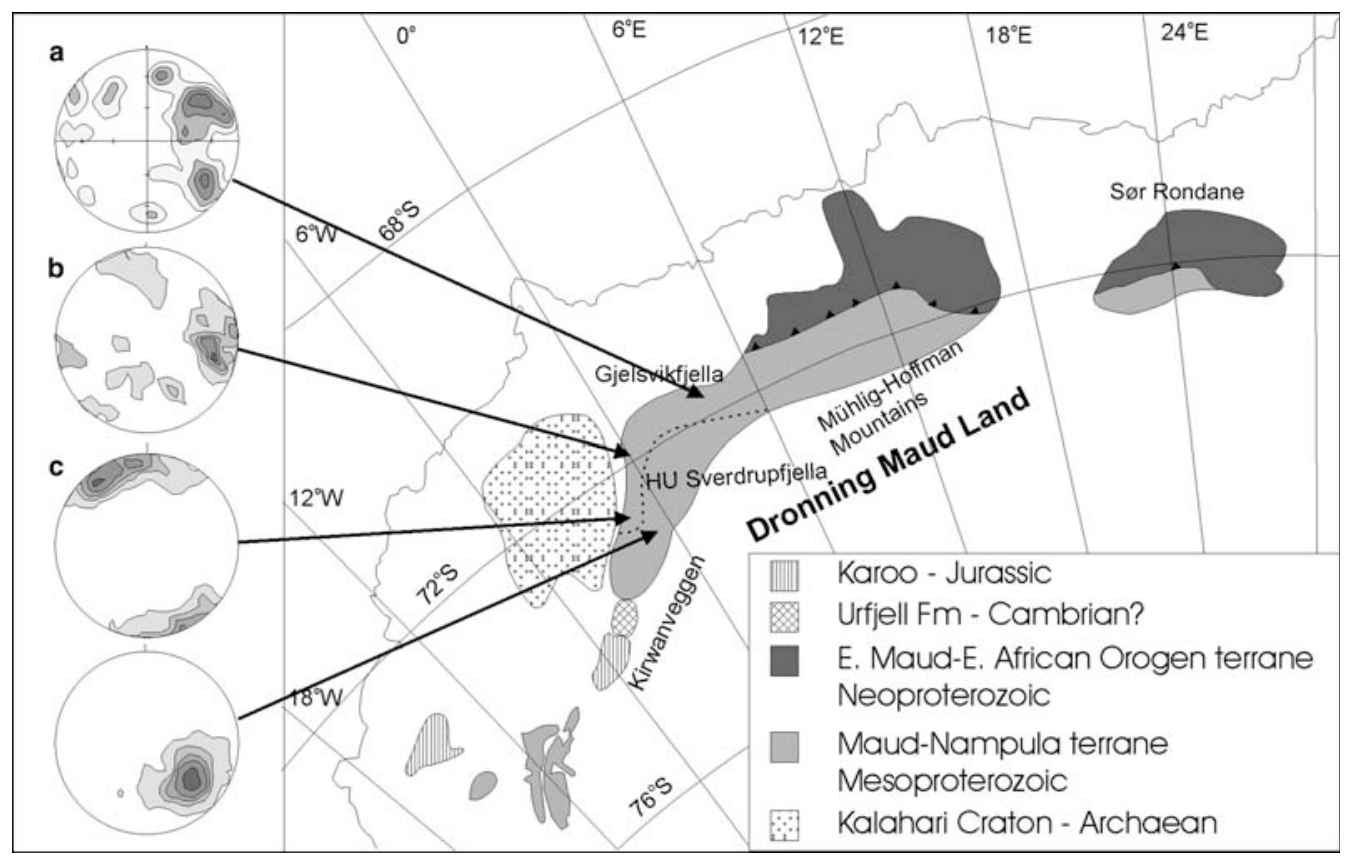

Fig. 8. Stereographic projections of lineations from various localities in western Dronning Maud Land.

granitic magmatism at c. $6 \mathrm{kbar}$ and $c .700{ }^{\circ} \mathrm{C}$ at $c$. $490 \mathrm{Ma}$ (Grantham et al. 1991). Progressing southward in Sverdrupfjella to Kirwanveggen, $P-T$ conditions at Neuemayerskarvet in the north of Kirwanveggen (Fig. 10h) described by Grantham et al. (2001) are of the order of $c$. $6.5 \mathrm{kbar}$ and c. $700{ }^{\circ}$ C. Significantly, c. $500 \mathrm{Ma}$ granitoids are absent in northern Kirwanveggen, and the $P-T$ estimates described by Grantham et al. (2001) were based on a thermally driven dehydration reaction of $\mathrm{Hbl}+\mathrm{Pl}+\mathrm{Qtz} \rightarrow \mathrm{Grt}+\mathrm{Ab}+\mathrm{H}_{2} \mathrm{O}$. Progressing southwards to southern Kirwanveggen, at Drapane, c. $530 \mathrm{Ma}$ sandstones and grits of the Urfjell Group are exposed, implying that at $c$. $530 \mathrm{Ma}$ the southern Kirwanveggen was exposed at surface. These data imply a crustal depth gradient between north and south Kirwanveggen of $c .6 \mathrm{kbar}$ or $c .20 \mathrm{~km}$.

The most important and fundamental difference between these various terranes is that in those areas with Namuno Block age signatures (Mugeba, Monapo, Highland Complex of Sri Lanka, NE Sør Rondane and Schirmacher Hills) the $P-T$ evolution at c. $550 \mathrm{Ma}$ is interpreted as involving significant isobaric cooling. In contrast, rocks with Nampula Block age signatures (Nampula, Sverdrupfjella, northern Kirwanveggen, Gjelsvikfjella and western Mühlig-Hofmannfjella and southwestern Sør Rondane) are largely characterized by extensive magmatism at 500-550 Ma, implying thermal heating. This aspect has been recognized by Baba et al. (2008).

\section{Discussion and conclusions}

This integrated study of geochronological and structural data and metamorphic $P-T$ paths supports an interpretation that rocks north of the Lurio Belt have been thrust southwards over the Nampula (Mozambique)-Maud (Antarctica) block as summarized in Figure 11. Figure 11 represents a schematic cross-section from northern Mozambique to southern Kirwanveggen with the staggered horizontal line representing current exposure levels in Africa and Antarctica superimposed on the inferred 600-500 Ma topographic profile. At the northern end, the Geci Group sediments were deposited at c. $580 \mathrm{Ma}$ (Melezhik et al. 2006; Fig. 2). Progressing southward, metamorphic conditions increase toward the Lurio Belt, with the Namuno Block being thrust over the Nampula Block. In the footwall, depression to greater depths resulted in thermal increase and partial melting, resulting in anatexis and granite genesis in the Nampula Block in Mozambique and in western DML, Antarctica. In this context, the numerical modelling of crustal melting in continental collision zones by England \& Thompson (1986) is applicable; they modelled the 

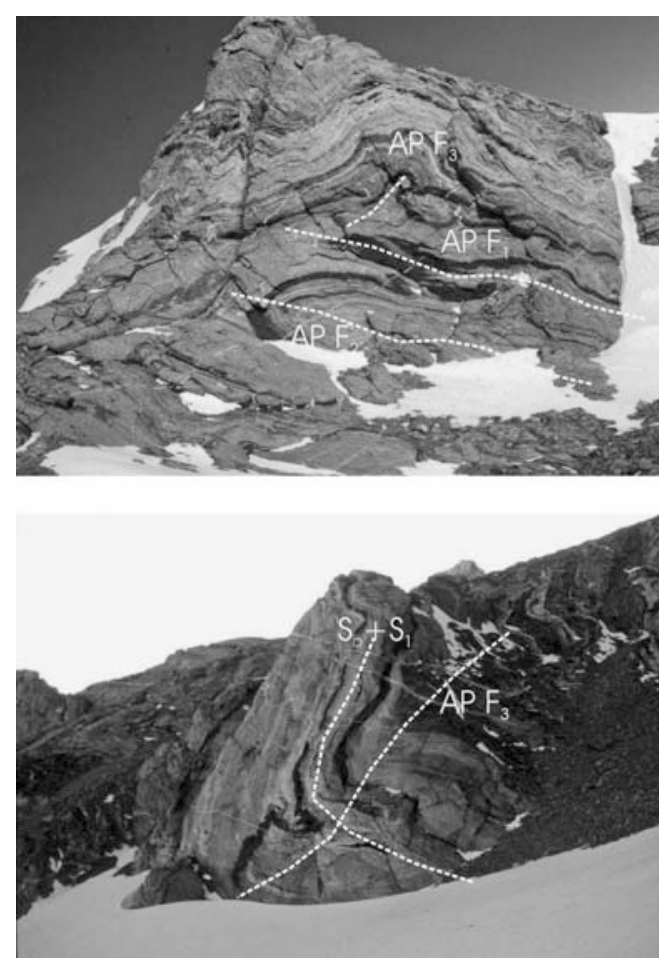

Fig. 9. Field photographs from Jutulrora, Sverdrupfjella, western DML. The upper photograph shows a

$\mathrm{NW}$-vergent $\mathrm{F}_{1}$ isoclinal recumbent fold with axial planar (AP) foliation, a NW-vergent $\mathrm{F}_{2}$ isoclinal fold in which the banding is clearly folded and a small SE-vergent $\mathrm{F}_{3}$ concentric fold. The lower photograph shows a larger-scale SE-vergent $\mathrm{F}_{3}$ fold with $\mathrm{NW}$-dipping axial plane. (Note also the SE-dipping thin granitic sheets in the lower photograph.)

$P-T$ evolution in a setting where crustal thickness is doubled by large-scale thrust faulting. Their model predicts that anatexis in the footwall would result in granite genesis $c$. $40 \mathrm{Ma}$ after the thrust-related thickening, depending on the level of anatexis. This modelling provides a plausible explanation of why the $c$. 530-495 Ma granites appear to be mostly undeformed and younger than the metamorphic ages, which start at $c .590 \mathrm{Ma}$. Following the thrust-related crustal thickening, isostatic rebound with associated inversion and extensional collapse would follow, with concomitant genesis and intrusion of the granites and pegmatites. The extensional structures resulting from the collapse of the orogenic pile would be oriented at $c .90^{\circ}$ to the $\sigma_{3}$ direction of the orogenic compression, as demonstrated in the Himalayas (Dewey 1988). Undeformed pegmatite dykes in the Nampula Block south of the Lurio Belt are correlated with the granites and have strikes dominantly toward the NNW, implying ENEWSW extension (Grantham et al. 2007b).

The section from northern to southern Kirwanveggen represents a progression from midcrustal levels to the surface, with the deposition of the Urfjell Group at c. $530 \mathrm{Ma}$. Figure 11 also shows that the $c$. 550-580 Ma top-to-the-SE deformation was superimposed on an older top-tothe-NW deformation recognized in western DML. The reorientation of structures immediately below the suture zone in the footwall is shown in Figure 11 and is consistent with the structures shown in Figure 9.

The interpretation of a large-scale thrust of East African Orogen rocks onto Antarctica has already been proposed by Ravikant et al. (2004, 2008), although their study did not examine the structural and metamorphic details. The interpretation presented in Figure 10 requires different levels of erosion between Africa and Sri Lanka and Antarctica. This difference in level of erosion is supported by the klippen of granulite remnants at Mugeba and Monapo on top of the Nampula Block footwall, as well as similar klippen remnants also preserved at Kataragama in Sri Lanka (Kriegsman, 1995, amongst others) (Fig. 4). Additional klippen along the northern Kalahari Craton margin that have similar geochronology (where data are available) include the Naukluft Mountains in Namibia (Ahrendt et al. 1978; Gray et al. 2006), the Urungwe Klippen in northern Zimbabwe (Shackleton et al. 1966), the Makuti Group (Dirks et al. 1999) and the allochthonous Masoso Suite (Dirks \& Jelsma 2006). Within the Zambezi Belt, the main phase of deformation involved transcurrent shearing and SW-vergent thrusting (Hanson et al. 1994; Wilson et al. 1997).

In contrast, Antarctica has not been subject to the same level of erosion, resulting in the preservation of a largely continuous slab of East African Orogen rocks from the nunataks at Mramornye c. $71^{\circ} \mathrm{S}$, c. $8^{\circ} \mathrm{E}$ (Piazolo 2004) via Schirmacher Oasis (Ravikant et al. 2002, 2004, 2008; Baba et al. 2006, 2008), the eastern Mühlig-Hofman mountains, NE Sør Rondane and beyond. We have not explored detailed aspects of the geology east of Sør Rondane other than to note that we are not aware of any rocks, east of SW Sør Rondane, with Nampula Block type lithological, geochronological and metamorphic characteristics. This may suggest that it is possible that rocks of the Nampula Terrane do not extend beyond the SW Sør Rondane.

Using the broad geographical grid references in Figure 4, the width of the overthrust block approximates to $4-5^{\circ}$ of latitude, equivalent to c. 480-600 km, representing the distance from the Lurio Belt in Mozambique to the sheared boundary 

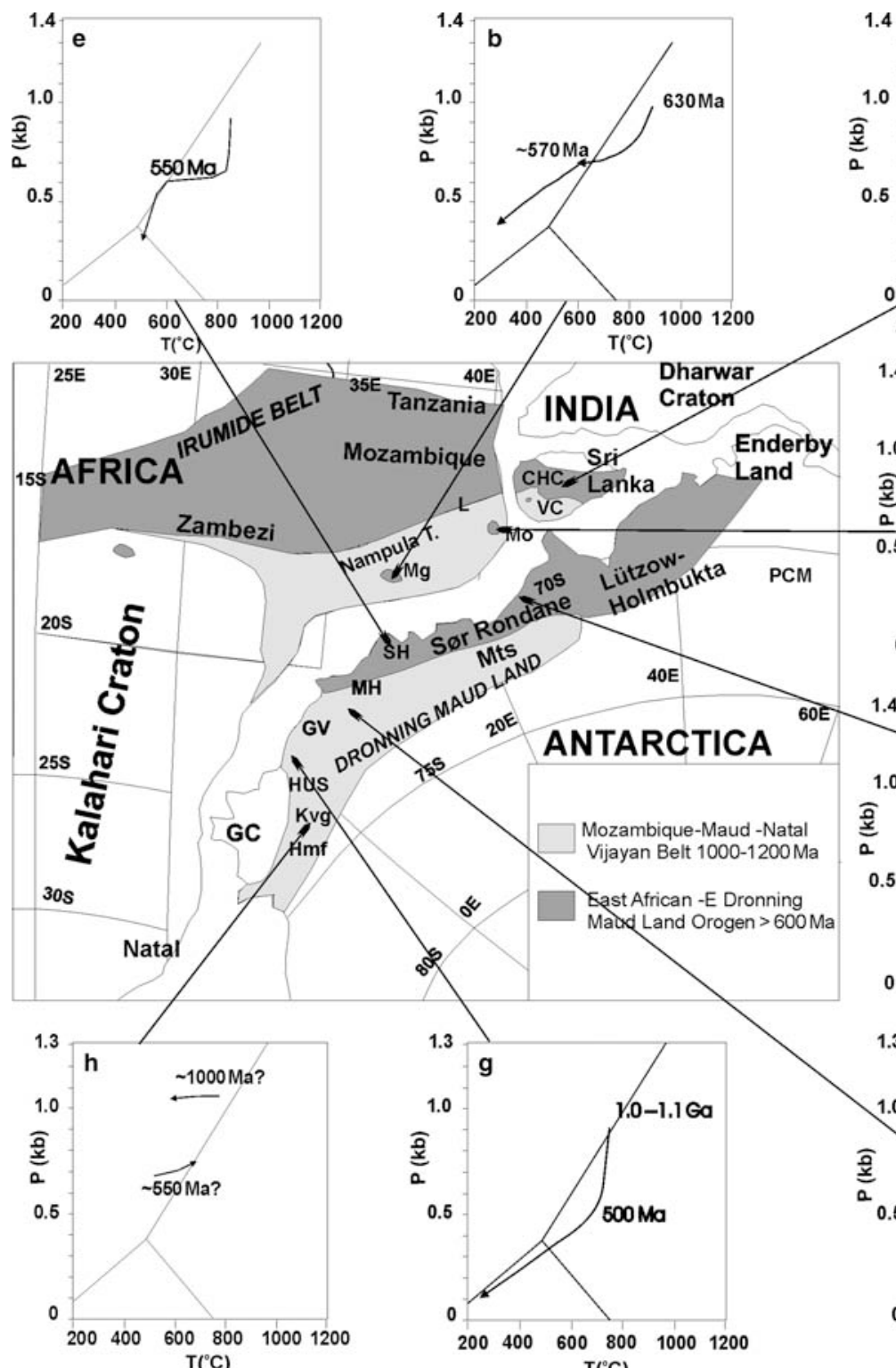

$\mathrm{T}\left({ }^{\circ} \mathrm{C}\right)$

Fig. 10. Figure summarizing $P-T$ loops from various localities in Mozambique, Sri Lanka, central DML, western DML and Sør Rondane as shown by the arrows linking the $P-T$ loops with the geographical locality. Abbrevations as in Figure 4.

between the two crustal blocks in Sør Rondane and Mühlig-Hofmannfjella. At the western extremity of the proposed belt, Martin (1974) estimated that the c. 560-570 Ma (Gray et al. 2006) Naukluft nappes had been transported at least $60 \mathrm{~km}$ towards the SE onto the Kalahari Craton.

For purposes of comparison, it should be noted that recent studies on the collision zone between
Peninsular India and Asia in the Himalayan Orogen have proposed crustal shortening of $c$. $550 \mathrm{~km}$ (Ratsbacher et al. 1994). If the crustal shortening had begun at $c .590 \mathrm{Ma}$ as suggested by the metamorphic ages in the Nampula Block, at a plate movement rate of $c .4 \mathrm{~cm} \mathrm{a}^{-1}$, similar to that recorded in current studies of the Himalayas (Ratsbacher et al. 1994), the emplacement of the 


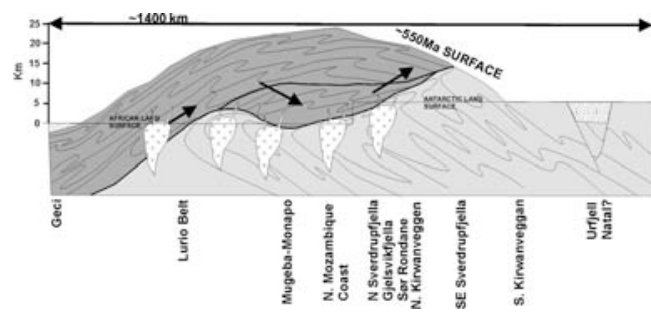

Fig. 11. Schematic cross-section representing the geological relationships from northern Mozambique to southern Kirwanveggen, Antarctica. The cross-section summarizes the deformational structures, the distribution of the 495-530 Ma granitic intrusions in the footwall resulting from burial heating, and the setting for the isothermal decompression followed by isobaric cooling paths of the hanging-wall Namuno Block. The cross-section also shows the relative difference in erosion levels between Africa and Antarctica.

mega-nappe would have required $c .15 \mathrm{Ma}$ to cover the possible $600 \mathrm{~km}$ envisaged. The nappe emplacement would then have been followed by inversion, isostatic uplift and erosion, with anatexis at depth c. $40 \mathrm{Ma}$ after emplacement of the obducted slab, as suggested by England \& Thompson (1986). The model of England \& Thompson (1986) demonstrates that it would require c. $100 \mathrm{Ma}$ for the thermal gradients of the footwall rocks to rise and achieve equilibrium with the hanging-wall rocks and that, depending on depth, it would take $c$. $40 \mathrm{Ma}$ for rocks at middle crustal level of the footwall to heat into the field of anatexis where melting would be initiated. The model also demonstrates that, depending on the relative depth of melting, one can generate a wide range of granitoids depending on whether the melts are 'minimum melts' or are produced by vapour-absent dehydration melting resulting in charnockitic K-rich anhydrous melts.

Although there appear to be different erosion levels in Africa and Antarctica, the implications of this model are that a substantial block of crust has been eroded and removed from this belt since c. $550 \mathrm{Ma}$, particularly in southern Africa. The recognition of reoriented fabrics in Sverdrupfjella and possibly northern Kirwanveggen suggests that these areas were probably in the footwall as well, providing an estimate of the area underlain by the nappe that is significantly larger than that reflected in Figure 4. The recognition of zircon populations with ages typical of the Namuno Block in the Transantarctic Mountains (Goodge 1997; Goodge et al. 2004) and similar rocks in Australia (Veevers et al. 2006) suggests that the sedimentary rocks now exposed in the Transantarctic Mountains and its extensions and the Ellsworth-Whitmore
Mountains (Flowerdew et al. 2007) were probably the depository of the erosion products from the collision of North and South Gondwana along the Damara-Zambezi-Lurio-Sri Lanka-central Dronning Maud axis. Another example of such deposition is in the Urfjell Group, in which the detrital zircon population (Fig. 12) has an age pattern unlike that of its surrounding Nampula Block type floor rocks (data from Croaker 1999) but a distribution more typical of the Namuno Block rocks from north of the Lurio Belt.

The overthrust block of tectonic units belonging to the Namuno Block as envisaged in this paper would have placed the detrital source for the Urfjell Group significantly closer to the depository and, in view of the age of the Urfjell Group of $c$. $530 \mathrm{Ma}$, the location of the Urfjell Group provides an absolute southern limit of the extent of the overthrust block. This model also provides insights into the geochronological differences between the East African Orogen (Stern 1994; Meert 2003) and the Kungu Orogeny proposed and described by Meert (2003). Stern (1994) initially described the northsouth-oriented East African Orogen based on fieldwork in North Africa, through Kenya and Tanzania and suggested that its timing was between $c$. $900 \mathrm{Ma}$ and c. $550 \mathrm{Ma}$. The model presented here suggests that, except for the structural outliers in the Nampula Block, Antarctica and Sri Lanka, the East African Orogen is terminated along the Lurio Belt, where it is overprinted and reworked by the Kungu Orogen. The collisional front along the Damara-Zambezi-Lurio-Sri Lanka-central Dronning Maud axis is therefore equivalent to the Kungu Orogen described by Meert (2003).

The rapid erosion and uplift of the Nampula Block is recorded in a titanite fission-track study by Daszinnies et al. (2006), who showed that the titanite fission-track ages north of the Lurio Belt

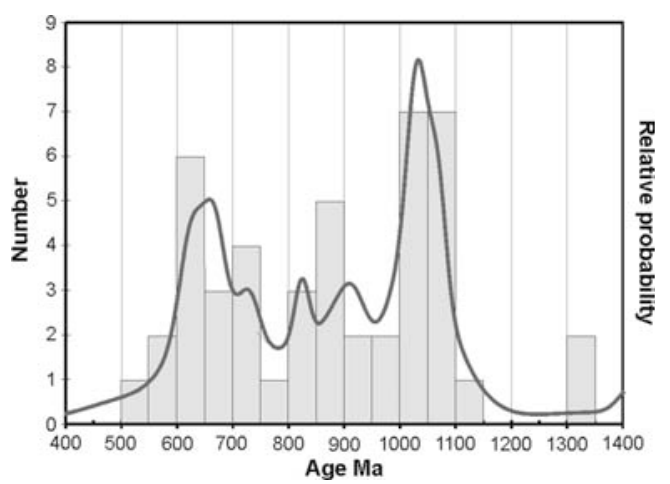

Fig. 12. Histogram and relative probability density curve of zircon ages from the Urfjell Group. Data from Croaker (1999). 
are $c .300 \mathrm{Ma}$ and become progressively younger to the coast, to $c$. $240 \mathrm{Ma}$. Erosion to surface was completed by $c$. $180 \mathrm{Ma}$, the age of the Karooage Angoche Andesite lavas on the northern Mozambique coast (Grantham et al. 2007b).

This model explains many of the correlation conundrums that have puzzled scientists in Gondwana reconstructions. It needs to be tested with much additional work. This work should probably involve constraining the precise ages of deformation in the vicinity of the suture zones between the Namuno type blocks and Nampula type blocks in Mozambique, Malawi, Sri Lanka and Antarctica. The extent of the overthrust blocks should also be tested with detailed geochronology and mapping on the Urungwe klippe in northern Zimbabwe, as well as geochronology and $P-T$ work on the nunataks at Mramornye in Dronning Maud Land. Another potential implication is that, after this collision, extensive parts of the northern Kalahari Craton may have been in the footwall of a large nappe. It is an open question as to whether there are other $>550 \mathrm{Ma}$ lithological units on the Zimbabwe Craton that may be allochthonous. Possible candidates include the Makuti Groups and the Rushinga Group in Zimbabwe and the Frontier Formation in Mozambique. The origin and distribution of the $c .850 \mathrm{Ma}$ rocks in Mozambique adjacent to those in the vicinity of the NE corner of Zimbabwe need to be studied in detail. Geochronology focusing on low-temperature closure systems in Zimbabwe may provide some idea of how far south the nappe travelled over Zimbabwe, if at all. Similarly, the grits, sandstones, shales and conglomerates of the pre-Karoo-age Sijarira Group in northwestern Zimbabwe may represent the Cambrian-age detritus eroded from the overthrust Zambezi Belt rocks and, if so, then their current positions constrain the southern limit of the mega-nappe (P. Dirks pers. comm.;), just as the Urfjell Group do in southern Kirwanveggen, western DML. A sedimentological study combined with a zircon provenance study of the Sijarira Group would be particularly illuminating. The uncertainty of the position of the southwestern end of the Lurio Belt in central northern Mozambique and southern Malawi also needs further fieldwork and geochronology.

We are sure that aspects of this model will be require revision and modification as new data are produced from further studies in Mozambique, Antarctica and Sri Lanka. New comprehensive geochronological and petrological data from Sri Lanka are vitally important. The model also needs to be tested with palaeomagnetic studies, particularly in view of the 'destruction', by crustal duplication followed by erosion, of large crustal blocks from the Namuno Block potentially involving $5^{\circ}$ of latitude or $600 \mathrm{~km}$.
We would like to acknowledge all the people who have over the years assisted in the field in various capacities, and who have assisted with data and sample collection in Antarctica and Mozambique. In Antarctica these include geologists R. Thomas and B. Groenewald, and in Mozambique these include R. Thomas, G. de Kock, M. du Toit, P. Botha, M. Kota, R. Opperman, M. Rohwer, J. C. Nolte, M. Cronwright, I. Haddon, J. Miller, S. De Azevedo, S. Fernando, R. Matola, G. Cune and S. Kagashima. We would also like to acknowledge the assistance of $\mathrm{H}$. Kaidan and D. Dunkley with SHRIMP analyses at NIPR, Tokyo. Critical reviews by R. Hansen and S. Johnson significantly improved the manuscript. Discussions with P. Dirks provided valuable insights into aspects related to the Zimbabwe Craton cover. Correspondence with C. Reeves led to the recognition that the Lurio Belt shear zone could also have a horizontal attitude. Permission to publish the summarized geochronological data from Mozambique was also granted by E. Daudi, Director of Direcão Nacional Geologia, Maputo, Mozambique. This paper is dedicated to the pioneers R. Sacchi and P. Pinna, who probably would have developed this model had they had the access to extensive single zircon geochronology.

\section{References}

Ahrendt, H., HunZiker, J. C. \& Weber, K. 1978. Age and degree of metamorphism and time of nappe emplacement along the southern margin of the Damara Orogen/Namibia. Geologische Rundschau, 67, 719-742.

AQUATER. 1983. Relatorio Final Vol. II-Cartografia Geologica. Cartografia Geologica e Prospecçao Mineira e Geoquimica nas Provincias de Nampula e da Zambezia.

Asami, M., Suzuki, K. \& GRew, E. S. 2005. Monazite and zircon dating by the chemical $\mathrm{Th}-\mathrm{U}-$ total $\mathrm{Pb}$ isochron method (CHIME) from Aleysheyev Bight to the Sør-Rondane Mountains, East Antarctica: A reconnaissance study of the Mozambique suture in Eastern Queen Maud Land. Journal of Geology, 113, 59-82.

BabA, S., OWADA, M., Grew, E. \& Shiraishi, K. 2006. Sapphirine-orthopyroxene-garnet granulite from Schirmacher Hills, Central Dronning Maud Land. In: Fütterer, D. K., Damaske, D., Kleinschmidt, G., Miller, H. \& Tessensohn, F. (eds) Antarctica: Contributions to Global Earth Sciences. Springer, New York, 37-44.

BabA, S., OWAdA, M. \& ShIRAishi, K. 2008. Contrasting metamorphic $P-T$ path between Schirmacher Hills and Mühlig-Hofmannfjella, Central Dronning Maud Land, East Antarctica. In: SATISH-KUMAR, M., Motoyoshi, Y., Osanai, Y., HiRoi, Y. \& ShIRAISHI, K. (eds) Geodynamic Evolution of East Antarctica: a Key to the East-West Gondwana Connection. Geological Society, London, Special Publications, 308, 401-418.

Barr, M. W. C. \& Brown, M. A. 1987. Precambrian gabbro-anorthosite complexes, Tete Province, Mozambique. Geological Journal, 22, 139-159. 
Barton, C. M., Evans, J. A., CARney, J. N. \& Crow, M. J. 1993. Geological and structural framework of the Zambezi Belt, northeastern Zimbabwe. In: Findlay, R. H., UnRUG, R., Banks, M. R. \& VeEvers, J. J. (eds) Gondwana Eight. Balkema, Rotterdam, 55-68.

BAuer, W., JACOBS, J. \& PAECH, H.-J. 2004. Structural evolution of the metamorphic basement of Central Dronning Maud Land, East Antarctica. Geologische Jahrbuch, B96, 325-363.

Baur, N., Kröner, A., Liew, T. C., Todt, W., Williams, I. S. \& HoffmanN, A. W. 1991. U-Pb isotopic systematics of zircons from prograde and retrograde transition zones in high-grade orthogneisses, Sri Lanka. Journal of Geology, 99, 527-545.

Bingen, B. I., Viola, G., Henderson, I. H. C. ET AL. 2006. Geochronology of Pan-African terrain assembly in NE Mozambique. In: Abstracts CAG 21, 3-5 July, Maputo, Mozambique, 12-14.

Bisnath, A. \& Frimmel, H. E. 2005. Metamorphic evolution of the Maud Belt: $P-T-t$ path for highgrade gneisses in Gjelsvikfjella, Dronning Maud Land, East Antarctica. Precambrian Research, 43 505-524.

Bisnath, A., Frimmel, H. E., Armstrong, R. A. \& BOARD, W. S. 2006. Tectonothermal evolution of the Maud Belt: new SHRIMP U-Pb zircon data from Gjelsvikfjella, Dronning Maud Land, East Antarctica. Precambrian Research, 150, 95-121.

Bjerkgard, T., Nordgulen, O., Bingen, B. I. ET AL. 2006. Overview of the Proterozoic geology in Niassa and Cabo Delgado Provinces, N. Mozambique. In: Abstracts CAG 21, 3-5 July, Maputo, Mozambique, $15-18$.

Board, W. S., Frimmel, H. E. \& Armstrong, R. A. 2005. Pan-African tectonism in the western Maud Belt: $P-T-t$ path for high-grade gneisses in the H.U. Sverdrupfjella, East Antarctica. Journal of Petrology, 46, 671-699.

Bonin, B. 2007. A-type granites and related rocks: Evolution of a concept, problems and prospects. Lithos, 97, 1-29.

Cadoppi, P., Costa, M. \& SACChi, R. 1987. A cross section of the Namama Thrust belt (Mozambique). Journal of African Earth Sciences, 6, 493-504.

Costa, M., CADoppi, P., SACChI, R. \& FAnNing, C. M. 1994. U-Pb SHRIMP dating of zircons from Mozambique Gneiss. Bolletino della Società Geologica Italiana, 113, 173-178.

CroAKer, M. 1999. Geological constraints on the evolution of the Urfjell Group, southern Kirwanveggen, western Dronning Maud Land, Antarctica. MSc thesis, University of Natal, Pietermaritzburg.

DAsZinnies, M. C. I., EMmel, B., JACOBS, J. \& Grantham, G. 2006. Implications for Gondwana rifting in Northern Mozambique: constrains from titanite and apatite fission track thermochronology. In: Abstracts CAG 21, 3-5 July, Maputo, Mozambique, 35-36.

DEWEY, J. F. 1988. Extensional collapse of orogens. Tectonics, 6, 1123-1139.

Dirks, P. H. G. M. \& Jelsma, H. A. 2006. The structural-metamorphic evolution of the northern margin of the Zimbabwe craton and the adjacent
Zambezi belt in northeastern Zimbabwe. In: Processes on the Early Earth. Geological Society of America, Special Papers, 405, 291-313.

Dirks, P. H. G. M., Jelsma, H. A., VinyU, M. L. \& Munyanyiwa, H. 1998. The structural history of the Zambezi Belt in northeast Zimbabwe; evidence for crustal extension during the early Pan-African. South African Journal of Geology, 101, 1-16.

Dirks, P. H. G. M., Kröner, A., Jelsma, H. A., Sithole, T. A. \& VinyU, M. L. 1999. Structural relations and $\mathrm{Pb}-\mathrm{Pb}$ zircon ages for the Makuti Gneisses: evidence for a crustal-scale Pan African shear zone in the Zambezi Belt, northwest Zimbabwe. Journal of African Earth Sciences, 28, 427-442.

EBY, G. N. 1992. Chemical subdivision of the A-type granitoids: Petrogenetic and tectonic implications. Geology, 20, 641-644.

England, P. C. \& Thompson, A. 1986. Some thermal and tectonic models for crustal melting in continental collision zones. In: CowARD, M. P. \& RiEs, A. C. (eds) Collision Tectonics. Geological Society, London, Special Publications, 19, 83-94.

Flowerdew, M. J., Millar, I. L., Curtis, M. L., Vaughan, A. P. M., Horstwood, M. S. A., Whitehouse, M. J. \& Fanning, C. M. 2007. Combined $\mathrm{U}-\mathrm{Pb}$ geochronology and $\mathrm{Hf}$ isotope geochemistry of detrital zircons from early Paleozoic sedimentary rocks, Ellsworth-Whitmore Mountains block, Antarctica. Geological Society of America Bulletin, 119, 275-288.

Goodge, J. 1997. Latest Neoproterozoic basin inversion of the Beardmore Group, central Transantarctic Mountains, Antarctica. Tectonics, 16, 682-701.

Goodge, J. W., Williams, I. S. \& Myrow, P. 2004. Provenance of Neoproterozoic and lower Paleozoic siliciclastic rocks of the central Ross orogen, Antarctica: Detrital record of rift-, passive-, and active-margin sedimentation. Geological Society of America Bulletin, 116, 1253-1279.

Grantham, G. H. 1992. Geological evolution of western H.U. Sverdrupfjella, Dronning Maud Land, Antarctica. $\mathrm{PhD}$ thesis, University of Natal, Pietermaritzburg.

Grantham, G. H., Moyes, A. B. \& Hunter, D. R. 1991. The age, petrogenesis and emplacement of the Dalmatian Granite, H. U. Sverdrupfjella, Dronning Maud Land, Antarctica. Antarctic Science, 3, 197-204.

Grantham, G. H., Jackson, C., Moyes, A. B., Groenewald, P. B., Harris, P. D., Ferrar, G. \& KRYNAUW, J. R. 1995. The tectonothermal evolution of the Kirwanveggan-H.U. Sverdrupfjella areas, Dronning Maud Land, Antarctica. Precambrian Research, 75, 209-230.

Grantham, G. H., Storey, B. C., Thomas, R. J. \& JACOBS, J. 1997. The pre-breakup position of Haag Nunataks within Gondwana: possible correlatives in Natal and Dronning Maud Land. In: RICCI, C. A. (ed.) The Antarctic Region: Geological Evolution and Processes. Terra Antartica, Sienna, $13-20$.

Grantham, G. H., Eglington, B. M., Thomas, R. J. \& Mendonidis, P. 2001. The nature of the Grenvilleage charnockitic A-type magmatism from the Natal, Namaqua and Maud Belts of southern Africa and western Dronning Maud Land, Antarctica. Memoirs 
of National Institute of Polar Research, Special Issue, $\mathbf{5 5}, 59-86$.

Grantham, G. H., Maboko, M. \& Eglington, B. M. 2003. A review of the evolution of the Mozambique Belt and implications for the amalgamation of Rodinia and Gondwana. In: YoshidA, M., Windley, B. F. \& Dasgupta, S. (eds) Proterozoic East Gondwana: Supercontinent Assembly and Breakup. Geological Society, London, Special Publications, 206, 401-426.

Grantham, G. H., Armstrong, R. A. \& Moyes, A. B. 2006. The age, chemistry and structure of mafic dykes at Roerkulten, H.U. Sverdrupfjella, western Dronning Maud Land, Antarctica. In: Hanski, E., Mertanen, S., Ramo, T. \& Vuollo, J. (eds) Dyke Swarms-Time Markers of Crustal Evolution. Proceedings of the Fifth/Fourth International Dyke Conference (IDC5), Rovaniemi, Finland. Balkema, Rotterdam, 213-224.

Grantham, G. H., Ingram, B. A., Cronwright, M. C. ET AL. 2007a. Map Explanation of Sheets Furancungo (1433) and Ulongue (1434). National Directorate of Geology, Republic of Mozambique, Maputo.

Grantham, G. H., Macey, P. H., IngRam, B. A. ET AL. 2007b. Map Explanation of Sheets Meconte (1439) and Nacala (1440). National Directorate of Geology, Republic of Mozambique, Maputo.

Gray, D. R., Foster, D. A., Goscombe, B., Passchier, C. W. \& Trouw, R. A. J. 2006. ${ }^{40} \mathrm{Ar} /{ }^{39} \mathrm{Ar}$ thermochronology of the Pan-African Damara Orogen, Namibia, with implications for tectonothermal and geodynamic evolution. Precambrian Research, 150, 49-72.

Groenewald, P. B., Moyes, A. B., Grantham, G. H. \& KRYNAUW, J. R. 1995. East Antarctic crustal evolution-geological constraints and modelling in western Dronning Maud Land. Precambrian Research, 75, 231-250.

HANSON, R. E., Wilson, T. J. \& MunyanyiWa, H. W. 1994. Geologic evolution of the Neoproterozoic Zambezi orogenic belt in Zambia. Journal of African Earth Sciences, 18, 135-150.

Hargrove, U. S., Hanson, R. E., Martin, M. W., Blenkinsop, T. G., Bowring, S. A., WALKer, N. \& Munyanyiwa, H. 2003. Tectonic evolution of the Zambezi orogenic belt: geochronological, structural, and petrological constraints from northern Zimbabwe. Precambrian Research, 123, 159-186.

HARRIS, P. D. 1999. The geological evolution of Neumayerskarvet in the northern Kirwanveggen, western Dronning Maud Land, Antarctica. PhD thesis, Rand Afrikaans University, Johannesburg.

Harris, P. D., Moyes, A. B., FAnning, C. M. \& ARMSTRONG, R. A. 1995. Zircon ion microprobe results from the Maudheim High Grade Gneiss Terrane, Western Dronning Maud Land, Antarctica. In: Geocongress '95 Abtracts, Rand Afrikaans University, Johannesburg, 240-243.

HiroI, Y., OGO, Y. \& NAMB A, K. 1994. Evidence for prograde metamorphic evolution of Sri Lankan pelitic granulites, and implications for the development of continental crust. Precambrian Research, 66, 245-263.

Hollick, L., Bauer, W., De Sousa SoARes, H. ET AL. 2006. Structural evolution in N. Mozambique-Milange, Mocuba and Insaca-Gurue Sheets. In: Abstracts CAG 21, 3-5 July, Maputo, Mozambique, 68-70.
Holzl, S., Hofmann, A. W., Todt, W. \& Kohler, H. 1994. U-Pb geochronology of the Sri Lankan basement. Precambrian Research, 66, 123-149.

JACKSON, C. 1999. Characterisation of Mesoproterozoic to Paleoozoic crustal evolution of western Dronning Maud land. Unpublished report to South African National Antarctic Programme. Study 3.

JACKSON, C. \& ARMSTROnG, R. A. 1997. The tectonic evolution of the central Kirwanveggen, Dronning Maud Land, Antarctica: Temporal resolution of deformation episodes using SHRIMP $\mathrm{U}-\mathrm{Pb}$ zircon geochronology. In: Tectonics Division of the Geological Society of South Africa, 13th Anniversary Conference, Abstracts, 21-22.

Jacobs, J., Fanning, C. M., Henjes-Kunst, F., Olesch, M. \& PAECH, H. J. 1998. Continuation of the Mozambique Belt into East Antarctica: Grenville age metamorphism and polyphase Pan-African high grade events in Central Dronning Maud Land. Journal of Geology, 106, 385-406.

JACOBS, J., BAUER, W. \& FANNInG, C. M. 2003a. Late Neoproterozoic/Early Paleozoic events in central Dronning Maud Land and significance for the southern extension of the East African Orogen into East Antarctica. Precambrian Research, 126, 27-53.

JACOBS, J., BAUER, W. \& FANNing, C. M. $2003 b$. New age constraints for Grenvillian age metamorphism in western central Dronning Maud land (East Antarctica) and implications for the paleogeography of Kalahari in Rodinia. Geologische Rundschau, 92, $301-315$.

JACOBS, J., KLemD, R., FAnNing, C. M., BAUeR, W. \& Colombo, F. 2003c. Extensional collapse of the late Neoproterozoic-early Paleozoic East AfricanAntarctic Orogen in Central Dronning Maud Land, East Antarctica. In: Yoshida, M., Windley, B. F. \& Dasgupta, S. (eds) Proterozoic East Gondwana: Supercontinent Assembly and Breakup. Geological Society, London, Special Publications, 206, 271-287.

JAMAL, D. L. 2005. Crustal studies across selected geotransects in NE Mozambique: Differentiating between Mozambiquan ( Nibaran) and Pan African events, with implications for Gondwana studies. $\mathrm{PhD}$ thesis, University of Cape Town.

Johnson, S. P., Rivers, T. \& DE WAele, B. 2005. A review of the Mesoproterozoic to early Palaeozoic magmatic and tectonothermal history of south-central Africa: implications for Rodinia and Gondwana. Journal of the Geological Society, London, 162, 433-450.

Johnson, S. P., DE WAele, B. \& Liyungu, K. A. 2006. $\mathrm{U}-\mathrm{Pb}$ sensitive high resolution ion microprobe (SHRIMP) zircon geochronology of granitoid rocks in eastern Zambia: Terrane subdivision of the Mesoproterozoic Southern Irumide Belt. Tectonics, 25, TC6004, doi:10.1029/2006TC001977.

Jourde, G. \& Vialette, Y. 1980. La chaîne du Lurio (Nord Mozambique). Un témoin de l'existence de chaînes Kibariennes (800-1350 Ma) en Afrique Orientale. BRGM Internal Report.

KLEINSCHRODT, R. 1994. Large-scale thrusting in the lower crustal basement of Sri Lanka. Precambrian Research, 66, 39-57. 
Koistinen, T. J., Lehtonen, M. I., Cune, G. ET AL. 2006 Geological mapping east and north of the Archaean Zimbabwe Craton, NW Mozambique. In: Abstracts CAG 21, 3-5 July, Maputo, Mozambique, 92.

KRETZ, R. 1983. Symbols for rock-forming minerals. American Mineralogist, 68, 277-279.

Kriegsman, L. M. 1995. The Pan-African event in East Antarctica: a view from Sri Lanka and the Mozambique Belt. Precambrian Research, 75, 263-277.

KRÖNER, A. 1991. African linkage of Precambrian Sri Lanka. Geologische Rundschau, 80, 429-440.

KRÖNER, A. 2001. Du Toit Memorial Lecture 1999: The Mozambique belt of East Africa and Madagascar: significance of zircon and $\mathrm{Nd}$ model ages for Rodinia and Gondwana supercontinent formation and dispersal. South African Journal of Geology, 104, $151-166$.

Kröner, A., Williams, I. S., Compston, W., BAur, N., Vitanage, P. W. \& Perera, L. R. K. 1987. Zircon ion microprobe dating of high grade rocks in Sri Lanka. Journal of Geology, 95, 775-791.

Kröner, A., Jaeckel, P. \& Williams, I. S. 1994. $\mathrm{Pb}$-loss patterns in zircons from a high-grade metamorphic terrain as revealed by different dating methods: $\mathrm{U}-\mathrm{Pb}$ and $\mathrm{Pb}-\mathrm{Pb}$ for igneous and metamorphic zircons from northern Sri Lanka. Precambrian Research, 66, 151-181.

Kröner, A., SAcchi, R., Jaeckel, P. \& Costa, M. 1997. Kibaran magmatism and Pan African granulite metamorphism in northern Mozambique: single zircon ages and regional implications. Journal of African Earth Sciences, 25, 467-484.

Kröner, A., Willner, A. P., Hegner, E., Jaeckel, P. \& Nemchin, A. 2001. Single zircon ages, PT evolution and $\mathrm{Nd}$ isotopic systematics of high grade gneisses in southern Malawi and their bearing on the evolution of the Mozambique belt in southeastern Africa. Precambrian Research, 109, 257-291.

Krynauw, J. R. \& JACKSON, C. 1996. Geological evolution of western Dronning Maud Land within a Gondwana framework. South African National Antarctic Programme Final Report 1991-1996. Geology Subsection, 1-48.

Lawver, L. A., Gahagen, L. M. \& Dalziel, I. W. D. 1998. A tight fit early Mesozoic Gondwana, a plate reconstruction perspective. Memoirs of National Institute of Polar Research, Special Issue, 53, 214-229.

Macey, P. H., Ingram, B. A., Cronwright, M. S. ET AL. 2007. Map Explanation of Sheets Alto Molócuè (1537), Murrupula (1538), Nampula (1539), Mogincual (1540), Errego (1637), Gilé (1638) and Angoche (1639-40). National Directorate of Geology, Republic of Mozambique, Maputo.

Manhica, A. S. T. D., Grantham, G. H., Armstrong, R. A., Guise, P. G. \& Kruger, F. J. 2001. Polyphase deformation and metamorphism at the Kalahari Craton-Mozambique Belt boundary. In: Miller, J. A., Holdsworth, R. E., Buick, I. S. \& HAND, M. (eds) Continental Reactivation and Reworking. Geological Society, London, Special Publications, 184, 303-321.

MÄnttärRi, I., Koistinen, T., Lehtonen, M. I., Manninen, T., Makitie, H., Huhma, H. \& Kuosmanen, E. 2006. U-Pb and $\mathrm{Sm}-\mathrm{Nd}$ ages for 27 magmatic rocks, NW Mozambique. In: Abstracts CAG 21, 3-5 July, Maputo, Mozambique, 203-205.

Martin, H. 1974. Damara rocks as nappes on the Naukluft Mountains, South West Africa. Precambrian Research Unit, University of Cape Town, Bulletin, 15, $153-165$.

MeERT, J. 2003. A synopsis of events related to the assembly of eastern Gondwana. Tectonophysics, 362, $1-40$.

Melezhik, V. A., Kuznetsov, A. B., Fallick, A. E., Smith, R. A., Gorokhov, I. M., Jamal, D. \& Catuane, F. 2006. Depositional environments and an apparent age for the Geci meta-limestones: Constraints on the geological history of northern Mozambique. Precambrian Research, 148, 19-31.

Moyes, A. B. \& HARRIS, P. D. 1996. Geological Evolution of Western Dronning Maud Land within a Gondwana Framework. South African National Antarctic Programme Final Report 1991-1996. Radiogenic Isotope Project, 1-38.

Moyes, A. B., KNoper, M. W. \& Harris, P. D. 1997. The age and significance of the Urfjell Group, western Dronning Maud Land. In: RicCI, C. A. (ed.) The Antarctic Region: Geological Evolution and Processes. Terra Antartica, Sienna, 31-36.

Paulsson, O. \& Austrheim, H. 2003. A geochronological and geochemical study of rocks from Gjelsvikfjella, Dronning Maud Land, Antarcticaimplications for Mesoproterozoic correlations and assembly of Gondwana. Precambrian Research, 125, $113-138$.

PiAzolo, S. 2004. Detailed geological mapping of the Mramornye nunataks and the western part of Schirmacheroase, Central Dronning Maud Land, East Antarctica. Geologische Jahrbuch, B96, 469-497.

PinNA, P. 1995. On the dual nature of the Mozambique Belt, Mozambique to Kenya. Journal of African Earth Sciences, 21, 477-480.

Pinna, P. \& Marteau, P. 1987. Carta geologica de Moçambique, 1:1000 000 scale, with explanatory notes. Instituto Naçional de Geologica, Maputo.

Pinna, P., Jourde, G., Calvez, J. Y., Mroz, J. P. \& MARQues, J. M. 1993. The Mozambique Belt in northern Mozambique: Neoproterozoic (1100-850 Ma) crustal growth and tectogenesis, and superimposed Pan-African (800-550 Ma) tectonism. Precambrian Research, 62, 1-59.

RAASe, P. \& SchenK, V. 1994. Petrology of granulitefacies metapelites of the Highland Complex, Sri Lanka: implications for the metamorphic zonation and the $P-T$ path. Precambrian Research, 66, $265-294$.

Ratsb ACher, L., Frisch, W., LiU, G. \& Chen, C. 1994. Distributed deformation in southern and western Tibet during and after the India-Asia collision. Journal of Geophysical Research, 99, 19917-19946.

Ravikant, V., Bhaskar Rao, Y. J. \& Gopalan, K. 2002. $P-T-t$ history of granulites from the Schirmacher Oasis, East Antarctica. 12th Annual V. M. Goldschmidt Conference Abstracts. Geochimica et Cosmochimica Acta, 66, 793.

RaVikant, V., Bhaskar RaO, Y. J. \& Gopalan, K. 2004. Schirmacher Oasis as an extension of the 
Neoproterozoic East African Orogen into Antarctica: New Sm-Nd isochron age constraints. Journal of Geology, 112, 607-616.

Ravikant, V., LauX, J. H. \& Pimentel, M. 2008. Sm$\mathrm{Nd}$ and $\mathrm{U}-\mathrm{Pb}$ isotopic constraints for crustal evolution during Late Neoproterozoic from rocks of the Schirmacher Oasis, East Antarctica: geodynamic development coeval with the East African Orogeny. US Geological Survey, Open-File Report 2007-1047, Geological Survey and the National Academies, USES OF-2007-1047, Short Research Paper 007; doi:10.3133/of2007-1047.srp007.

ReEves, C. \& DE WIT, M. 2000. Making ends meet in Gondwana: retracing the transforms of the Indian Ocean and reconnecting continental shear zones. Tectonics, 12, 272-280.

Roberts, M. P., Grantham, G. H., Cronwright, M. ET AL. 2005. Preliminary pressure temperature determinations on granulite-facies rocks of the Mugeba Klippe, north central Moçambique. In: GEO2005 Extended Abstracts, Durban, South Africa, 193-194.

Sacchi, R., Marques, J., Costa, M. \& Casati, C. 1984. Kibaran events in the southern Mozambique Belt. Precambrian Research, 25, 141-159.

SACCHI, R., CAdoppi, P. \& Costa, M. 2000. Pan-African reactivation of the Lurio segment of the Kibaran Belt system: a reappraisal from recent age determinations in northern Mozambique. Journal of African Earth Sciences, 30, 629-639.

Schumacher, R., Schenk, V., RAase, P. \& Vitanage, P. W. 1990. Granulite facies metamorphism of metabasic and intermediate rocks in the Highland series of Sri Lanka. In: AshworTH, J. R. \& Brown, M. (eds) High grade Metamorphism and Crustal Anatexis. Unwin Hyman, Boston, MA, 235-271.

Shackleton, R. M., VAil, J. R. \& Wood, D. S. 1966. Preliminary report on the origin and significance of the Urungwe Klippe, Rhodesia. Annual Report, Research Institute of African Geology, University of Leeds, $\mathbf{1 0}$, 10-13.

ShIRAISHI, K. \& KAGAMI, H. 1992. Sm-Nd and Rb-Sr ages of metamorphic rocks from the Sør Rondane Mountains, east Antarctica. In: YoshidA, Y., Kaminuma, K. \& Shiraishi, K. (ed.) Recent Progress in Antarctic Earth Science. Terra, Tokyo, 29-35.

Shiraishi, K., AsAmi, M., IshizUKA, H. ET AL. 1991. Geology and metamorphism of the Sør Rondane mountains, east Antarctica. In: Thomson, M. R. A., Crame, J. A. \& Thomson, J. W. (eds) Geological Evolution of Antarctica. Cambridge University Press, Cambridge, 77-82.

Shiraishi, K., Dunkley, D., Hokada, T., FAnning, C. M., Kagami, H. \& Hamamoto, T. 2008. Geochronological constraints of the Late Proterozoic-Cambrian crustal evolution in eastern Dronning Maud Land, East Antarctica: a synthesis of SHRIMP $\mathrm{U}-\mathrm{Pb}$ age and $\mathrm{Nd}$ model age data. In: SATISH-KUMAR, M., Motoyoshi, Y., Osanai, Y., Hiroi, Y. \& ShIRAISHI, K. (eds) Geodynamic Evolution of East Antarctica: a Key to the East-West Gondwana Connection. Geological Society, London, Special Publications, 308, 21-68.

Siegfried, P. R. 1999. The Monapo structure and intrusive complex-An example of large scale alkaline metasomatism in northern Mozambique. In: Stanley, C. J. ET AL. (eds) Mineral Deposits: Processes to Processing. Balkema, Rotterdam, 683-686.

Sircombe, K. 2000. The usefulness and limitations of binned frequency histograms and probability density distributions for displaying absolute age data. Radiogenic age and isotopic studies. Geological Survey of Canada, Report, 13.

STERN, R. J. 1994. Arc assembly and continental collison in the Neoproterozoic East African Orogen: Implications for the consolidation of Gondwanaland. Annual Review of Earth Science, 22, 319-351.

Thomas, R. J., Bauer, W., Bingen, B. ET AL. 2006. Mozambique Belt in the Milange-MocubaMalemba area, Mozambique. In: Abstracts CAG 21, 3-5 July, Maputo, Mozambique, 161-163.

Veevers, J. J., Belousova, E. A., SAeed, A., Sircombe, K., Cooper, A. F. \& Read, S. E. 2006. Pan-Gondwanaland detrital zircons from Australia analysed for Hf-isotopes and trace elements reflect an ice-covered Antarctic provenance of 700-500 Ma age, $\mathrm{T}_{\mathrm{DM}}$ of $2.0-1.0 \mathrm{Ga}$, and alkaline affinity. Earth-Science Reviews, 76, 135-174.

Vinyu, M. L., Hansen, R. E., Martin, M. W., Bowring, S. A., Jelsma, H. A., Krol, M. A. \& DiRKs, P. H. G. M. 1999. U-Pb and ${ }^{40} \mathrm{Ar} /{ }^{39} \mathrm{Ar}$ geochronological constraints on the tectonic evolution of the eastern part of the Zambezi orogenic belt, northeast Zimbabwe. Precambrian Research, 98, 67-82.

Viola, G., Henderson, I., Bingen, B., Feitio, P., Thomas, R. J., Hollick, L. \& JacoBs, J. 2006. A new tectonic framework for northern Mozambique. In: Abstracts CAG 21, 3-5 July, Maputo, Mozambique, 168.

Wareham, C. D., Pankhurst, R. J., Thomas, R. J., Storey, B. C., Grantham, G. H., Jacobs, J. \& Eglington, B. M. 1998. Pb, Nd, and $\mathrm{Sr}$ isotope mapping of Grenville-age crustal provinces in Rodinia. Journal of Geology, 106, 647-659.

Westerhof, A. B. 2006. Tectonic framework of central and southern Mozambique in relation to Africa's major building blocks. In: Abstracts CAG 21, 3-5 July, Maputo, Mozambique, 174-177.

Wilson, T. J., Grunow, A. M. \& Hanson, R. E. 1997. Gondwana assembly: the view from southern Africa and East Gondwana. Journal of Geodynamics, 23, 263-268. 